\title{
VEGETACIÓN DE UN ÁREA POLDERIZADA DEL RÍO DE LA PLATA EN LA CIUDAD DE BUENOS AIRES: LA RESERVA ECOLÓGICA CIUDAD UNIVERSITARIA - COSTANERA NORTE
}

\author{
Rocío Melzi Fiorenza ${ }^{1,2}$, Horacio Sirolli ${ }^{1,2,3}$ \& Pablo I. Picca ${ }^{4}$
}

\begin{abstract}
${ }^{1}$ Departamento de Ecología, Genética y Evolución, Facultad de Ciencias Exactas y Naturales, Universidad de Buenos Aires, Intendente Güiraldes 2160, C1428EGA, Ciudad Autónoma de Buenos Aires, Argentina; rociomelzi@gmail.com (autora corresponsal).

${ }^{2}$ Instituto de Ecología, Genética y Evolución de Buenos Aires, Universidad de Buenos Aires - Consejo Nacional de Investigaciones Científicas y Técnicas, Intendente Güiraldes 2160, C1428EGA, Ciudad Autónoma de Buenos Aires, Argentina.

${ }^{3}$ Sector Conservación y Monitoreo, Reserva Ecológica Costanera Sur, Secretaría de Ambiente, Gobierno de la Ciudad Autónoma de Buenos Aires, Avenida Tristán Achával Rodríguez 1550, C1107ACR, Ciudad Autónoma de Buenos Aires, Argentina.

${ }^{4}$ Instituto de Micología y Botánica (CONICET-UBA), Departamento de Biodiversidad y Biología Experimental, Facultad de Ciencias Exactas y Naturales, Universidad de Buenos Aires, Intendente Güiraldes 2160, C1428EGA, Ciudad Autónoma de Buenos Aires, Argentina.
\end{abstract}

\begin{abstract}
Melzi Fiorenza, R.; H. Sirolli \& P. I. Picca. 2020. Vegetation of a Río de la Plata polderized area in Buenos Aires City: the "Ciudad Universitaria - Costanera Norte" Ecological Reserve. Darwiniana, nueva serie 8(2): 460-478.

Coastal reclamation constitutes a world trend and can result in new ecosystems adapted to the new coastal configuration. In Buenos Aires City, the "Ciudad Universitaria - Costanera Norte" Ecological Reserve (RECU-CN) constitutes one example of the expansion of the coastline in the Río de la Plata sector, carried out in the late 1960s. Given the lack of published works generated systematically that cover the entire area, we set as the objective to describe the RECU-CN vascular plant vegetation. For this, a systematic sampling was carried out in 79 plots of $25 \mathrm{~m}^{2}$, where the coverage of each of the vascular plant species present was registered. We identified 154 taxa, corresponding to 144 species, 123 genus and 58 families, and the total richness was estimated as 238 species. Trees and herbs had the highest coverage of all life forms. Through multivariate analysis, three vegetation units (Wetland, Forest and Grassland with groves) and 10 vascular plant communities were recognized. Vegetation was ordered according to two gradients: one of flood and another of anthropization. From the comparison with the typical plant communities of the region, similarities were found only with those registered in the Wetland unit. Results highlight that both the coexistence of physiognomically diverse vegetation units, as well as the native composition found in the plant communities of the Wetland unit, constitute main conservation values of the protected area.
\end{abstract}

Keywords. Coastal anthropogenic fill; Coastal wetland; Natural protected area; Novel ecosystem; polderization.

Resumen. Melzi Fiorenza, R.; H. Sirolli \& P. I. Picca. 2020. Vegetación de un área polderizada del Río de la Plata en la Ciudad de Buenos Aires: la Reserva Ecológica Ciudad Universitaria - Costanera Norte. Darwiniana, nueva serie 8(2): 460-478.

La expansión de la línea de costa por rellenos de origen antrópico constituye una tendencia mundial, pudiendo generar nuevos ecosistemas adaptados a la nueva configuración costera. En la ciudad de Buenos Aires, la Reserva Ecológica Ciudad Universitaria - Costanera Norte (RECU-CN) constituye un ejemplo de la expansión de la línea de costa en el Río de la Plata, realizada a fines de la década de 1960. Ante la falta de trabajos publicados generados en forma sistemática y que abarquen toda el área, se planteó como objetivo describir la vegetación de plantas vasculares de la RECU-CN. Para ello se realizó un muestreo sistemático en 79 parcelas de $25 \mathrm{~m}^{2}$, donde se relevó la cobertura de cada una de las especies de plantas vasculares presentes. Se registraron 154 taxones correspondientes a un total de 144 especies, 123 géneros y 58 familias y se estimó la riqueza total del área en 238 especies. Las formas de vida de mayor cobertura fueron los árboles y las hierbas. 
Mediante análisis multivariados se reconocieron tres unidades de vegetación (Humedal, Bosque y Pastizal con arboledas), y 10 comunidades de plantas vasculares. La vegetación se ordenó según dos gradientes: uno de inundación y otro de antropización. De la comparación con las comunidades de plantas típicas de la región, se encontraron similitudes sólo con aquellas registradas en la unidad de Humedal. Los resultados destacan que tanto la coexistencia de unidades de vegetación fisonómicamente diversas, como la presencia de comunidades nativas de la región en la unidad de Humedal constituyen valores de conservación importantes del área natural protegida.

Palabras clave. Área natural protegida; Humedal costero; Neoecosistema; polderización; Relleno costero.

\section{INTRODUCCIÓN}

Debido a la magnitud que han alcanzado los cambios producidos por el ser humano en la Tierra, los ecosistemas que se desarrollan espontáneamente, como respuesta natural a las nuevas condiciones ambientales creadas por la actividad humana son, desde una perspectiva ecológica, cada vez más relevantes (Lugo, 2009). Dichos ecosistemas, conocidos como neoecosistemas ("novel ecosystems"), resultan de la coocurrencia de especies en combinaciones y abundancias relativas que no se han verificado previamente para un dado bioma (Hobbs et al., 2006). En muchos casos, se trata de áreas en las que las especies exóticas invasoras son las dominantes o más frecuentes, mientras que las especies de plantas nativas se encuentran como acompañantes (Morello et al., 2000). Actualmente los neoecosistemas ocupan alrededor del $40 \%$ de la tierra libre de hielo, por lo que se han vuelto críticos para la conservación de la biodiversidad y la provisión de servicios ecosistémicos (Bridgewater et al., 2011). De este modo, es necesario entender estos sistemas para poder predecir su estado futuro y su correcto manejo adaptativo (Seastedt et al., 2008).

Las áreas naturales protegidas (ANP) son esenciales para la conservación de la diversidad biológica y el mantenimiento de un ambiente saludable para las personas (Naughton-Treves et al., 2005; UNEP-WCMC \& IUCN, 2016), siendo la biodiversidad local mayor dentro de las ANP que fuera de ellas (Gray et al., 2016), inclusive sin haberse desarrollado todo su potencial de conservación (Gaston et al., 2008; Watson et al., 2014). Muchas veces se han cuestionado las posturas de conservación que consideran a los ecosistemas urbanos, a menudo asociados a un gran número de especies no nativas.
Sin embargo, es prometedor considerar sus servicios ecosistémicos asociados, sus beneficios sociales y su contribución a la conservación de la diversidad biológica (Dearborn \& Kark, 2010; Kowarik, 2011). El establecimiento de las especies exóticas en ecosistemas urbanos está asociado a su cercanía a centros urbanos, tamaño y densidad poblacional humanos (Kuhman et al., 2010; Gaertner et al., 2017). En particular, las áreas naturales urbanas brindan la oportunidad de llevar a cabo tareas de conservación y de educación ambiental sobre los procesos ecosistémicos que allí tienen lugar. Además, promueven una mayor participación e involucramiento de los ciudadanos a través de la ciencia ciudadana, la restauración y el monitoreo ambiental (Dearborn \& Kark, 2010).

Las ANP de la costa bonaerense del Río de la Plata se encuentran declaradas tanto sobre ambientes remanentes como sobre sustratos jóvenes (Cabrera \& Dawson, 1944; Barbetti et al., 1985; Faggi \& Cagnoni, 1987; Kalesnik et al., 2005) y se encuentran influenciadas por tres regiones fitogeográficas distintivas: Delta del Paraná, Espinal y Pampeana (Burkart et al., 1999; Oyarzabal et al., 2018). Según Passarelli et al. (2014), en los humedales próximos al Río de la Plata pueden observarse comunidades herbáceas y leñosas de especies nativas, que incluyen también la presencia de algunas exóticas. Las ANP rioplatenses se encuentran, por un lado, insertas o muy próximas a centros urbanos muy densamente poblados y, por otro lado, próximos al Río de la Plata, cuyas márgenes constituyen un importante biocorredor que facilita el intercambio de fauna y propágulos vegetales (Capllonch et al., 2008; Guerrero et al., 2017).

La línea de costa de la ciudad de Buenos Aires ha sufrido modificaciones ambientales desde su primera fundación hace más de 500 años (Athor, 2012; Brailovsky, 2018). Su perfil es producto de sucesivos 
rellenos realizados desde el año 1836 sobre la planicie estuárica del Río de la Plata (Nabel \& Becerra Serial, 2008). Esta técnica de expansión de la costa por relleno es en la actualidad una tendencia mundial, mayormente mediada por factores antropogénicos, que tiende a la reducción del área ocupada por humedales costeros (Sengupta et al., 2018) y la pérdida de biodiversidad (Yang et al., 2011; Duan et al., 2016). En el caso particular de la costa rioplatense, en cada progradación la hidrodinámica natural del río fue generando nuevos ecosistemas adaptados a la configuración costera creada por los distintos sistemas de relleno (Marcomini \& López, 2004).

Uno de los últimos rellenos antrópicos sobre el Río de la Plata, realizado durante las décadas de 1960 y 1970, corresponde al terreno donde hoy se asienta la Reserva Ecológica Ciudad Universitaria - Costanera Norte (RECU-CN). Esta ANP fue creada mediante la Ley $\mathrm{N}^{\circ} 4467$ en el año 2012, pero no fue hasta marzo del año 2018, cuando se firmó el convenio entre los organismos que deben cogestionar el área (la Universidad de Buenos Aires y el Gobierno de la Ciudad Autónoma de Buenos Aires), que entró en vigencia dicha Ley. Para cumplir con los objetivos particulares de cualquier ANP es necesario contar con una línea de base de los recursos naturales del lugar, permitiendo así caracterizar los aspectos naturales y planificar el manejo de los mismos (APN, 2010). El área ha sido recorrida y utilizada con fines académicos desde sus orígenes, principalmente por docentes, investigadores y estudiantes de la Universidad de Buenos Aires. En cuanto a la vegetación del área, hasta el momento se realizó un listado preliminar de la flora vascular de un sector de 5 ha (Santo Domingo Sartori \& Picca, 2009) y una recopilación de la riqueza acumulada históricamente (Sirolli et al., 2018), pero no existen trabajos publicados generados en forma sistemática que abarquen toda el área. Por lo tanto, el objetivo del presente trabajo fue describir la vegetación de plantas vasculares de la RECU-CN mediante la identificación de gradientes ambientales en los que se ordena la vegetación y la clasificación de unidades homogéneas. Se planteó como hipótesis que las especies de plantas vasculares presentes en la RECU-CN se encuentran en combinaciones distintas a las de las comunidades de plantas naturales descriptas en la bibliografía de la región, conformándose como neoecosistemas.

\section{MATERIALES Y MÉTODOS}

\section{Área de estudio}

El área de estudio se encuentra al norte de la Ciudad Autónoma de Buenos Aires, dentro de la Comuna 13, a orillas del Río de la Plata, y abarca una superficie total de 23 ha (Fig. 1). Sus límites son el muro de contención de la Ciudad Universitaria, la desembocadura del arroyo White, el Río de la Plata y el Parque de la Memoria. En 1936 el predio ocupado actualmente por Ciudad Universitaria y la RECU-CN no existía, llegando el río hasta las vías del ferrocarril General Belgrano (Prezzi et al., 2011). Este terreno fue ocupado por rellenos sucesivos que ocuparon la plataforma de abrasión que afloraba en toda la costa de la ciudad, correspondiente al paleoacantilado que conformaban las barrancas de Belgrano y Nuñez (Prezzi et al., 2011). En particular, el área de estudio corresponde a un proceso parcial de polderización, realizado mediante la construcción de un terraplén unido a la costa sólo en un extremo, que delimitó una península y un humedal costero estuárico conectado al río por un canal de mareas ubicado al norte del área (Marcomini \& López, 2004). El clima es subtropical húmedo con una temperatura media anual de $17,9^{\circ} \mathrm{C}$ y una precipitación anual de 1082 mm (estación Aeroparque Jorge Newbery, Servicio Meteorológico Nacional, 2020). El área es afectada por el nivel del río, dominado mayormente por los vientos, dándose fenómenos climatológicos como las sudestadas. Este fenómeno se presenta como vientos provenientes del sector sudeste con persistencia regular e intensidades de moderadas a fuertes, que producen inundaciones en la costa del Río de la Plata con duraciones de uno a tres días (Kreimer et al., 2001).

\section{Obtención de datos}

El tamaño de la parcela se definió en 5 x $5 \mathrm{~m}$ $\left(25 \mathrm{~m}^{2}\right)$ luego de realizar un muestreo piloto en ocho puntos seleccionados al azar procurando tener en cuenta las distintas fisonomías vegetales detectadas “a priori” (comunidades herbáceas, arbóreas, terrestres y acuáticas). En cada punto se contabilizó el número de especies presentes en tamaños de subparcelas crecientes desde $0,3 \mathrm{x}$ $0,3 \mathrm{~m}$ hasta $10 \times 10 \mathrm{~m}$ en saltos que duplicaban la superficie de la parcela anterior, y se estableció el 


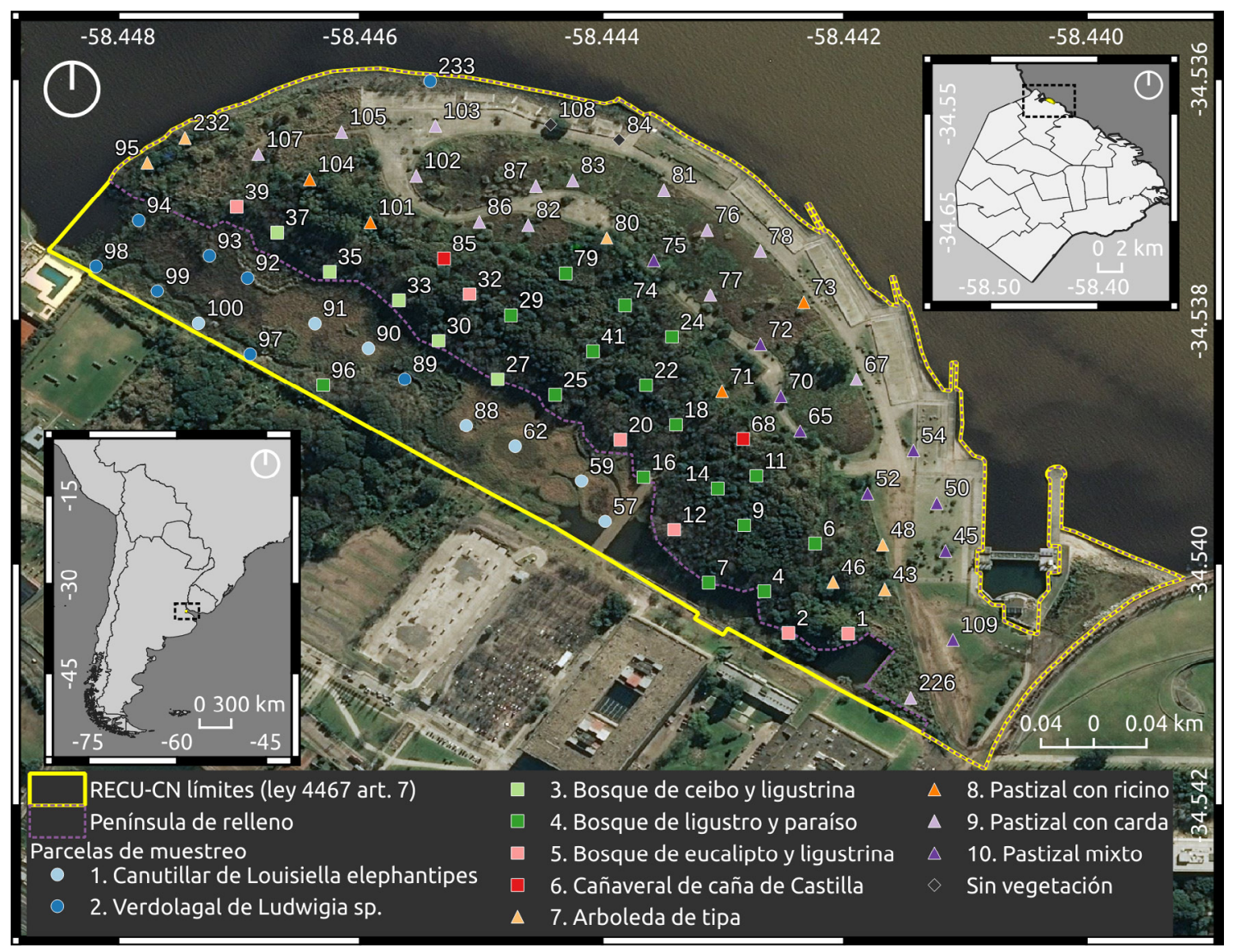

Fig. 1. Ubicación de la Reserva Ecológica Ciudad Universitaria - Costanera Norte (RECU-CN). Se indican los límites de la RECU-CN (línea amarilla), la ubicación de las parcelas de muestreo (números, no consecutivos), las unidades de vegetación Humedal (círculos), Bosque (cuadrados) y Pastizal con arboledas (triángulos) y las 10 comunidades de plantas (distintos colores, caña de castilla = Arundo donax, carda = Dipsacus fullonum, ceibo = Erythrina crista-galli, eucalipto = Eucalyptus sp., ligustrina $=$ Ligustrum sinense, ligustro $=$ L. lucidum, ricino $=$ Ricinus communis, tipa $=$ Tipuana tipu . Figura en color en la versión en línea http://www.ojs.darwin.edu.ar/index.php/darwiniana/article/view/908/1194

tamaño óptimo por la determinación del punto de inflexión de la curva de acumulación de especies (Mueller-Dombois \& Ellenberg, 1974). Una vez determinado el tamaño de parcela óptimo se procedió con el muestreo, disponiendo las parcelas de manera sistemática en toda el área de estudio en las intersecciones de una grilla de 50 x $50 \mathrm{~m}$ (Fig. 1). En total se estudiaron 79 parcelas, correspondiente a una superficie de 0,1975 ha, de las cuales dos no fueron consideradas por estar cubiertas sólo por hormigón. El muestreo se realizó de junio a agosto de 2018 durante un total de 14 jornadas, incluidas aquellas correspondientes al muestreo piloto.
La cobertura de cada especie se estimó visualmente mediante el método de Braun-Blanquet considerando las siguientes nueve categorías de rangos porcentuales $0-0,1 ; 0,1-1 ; 1-5 ; 5-10 ; 10$ $25 ; 25-33 ; 33-50 ; 50-75$ y 75-100 (van der Maarel \& Franklin, 2013). Aquellos ejemplares cuya identificación no pudo ser resuelta a campo fueron herborizados para su posterior determinación en el laboratorio. Para la determinación de los materiales se consultaron las floras regionales de Buenos Aires (Cabrera, 1963, 1965a, 1965b, 1967, 1968, 1970) y de Entre Ríos (Burkart, 1969, 1974, 1979, 1987; Burkart \& Bacigalupo 2005). 
De los 154 taxones registrados, 124 fueron identificados a nivel de especie, 20 a nivel de género y 10 a nivel de familia u otra jerarquía. Algunas especies no pudieron ser determinadas y son tratadas como morfotipos. La nomenclatura de las especies empleada en este trabajo siguió el criterio de Zuloaga et al. (2019), disponible en línea en el sitio web del Instituto de Botánica Darwinion (http://conosur.floraargentina.edu.ar/ [consultado marzo-noviembre 2019]). En la Tabla 1 se detalla la lista completa de especies con sus autores.

\section{Análisis de datos}

A escala global, de unidad de vegetación y de comunidad se calculó la riqueza como el número acumulado de taxones. Para cada taxón, la cobertura media correspondió al promedio de las coberturas registradas en las parcelas correspondientes, y la frecuencia relativa al total de parcelas en las que el taxón estuvo presente respecto al total de parcelas consideradas. Ambos valores se calcularon tanto para las unidades de vegetación como para las comunidades. Para la cobertura se utilizó la marca de clase de los rangos porcentuales utilizados. La riqueza total del área se estimó con la función $i N E X T$ del paquete $i N E X T$ (Hsieh et al., 2016) en R (R Core Team, 2019) a partir de la matriz de incidencia de los datos recolectados a campo (77 parcelas y 154 taxones). La riqueza, cobertura y frecuencia también fueron analizadas agrupando los taxones según su origen cuando pudo ser determinado (nativo o exótico) y su forma de vida (árbol, arbusto, subarbusto, hierba y trepadora).

Se realizaron análisis multivariados de la vegetación de la RECU-CN mediante R (R Core Team, 2019), previa transformación de los datos de cobertura a escala ordinal (1 a 9 para las nueve categorías porcentuales antes mencionadas, cero para ausencia). Dicha transformación se realizó con el objetivo de disminuir el riesgo de sobreenfatizar los taxones dominantes (Jensen 1978) y de corregir en forma aceptable los valores de cobertura para su análisis numérico (van der Maarel, 2007). La similitud en la composición de taxones de las parcelas y los gradientes ambientales en los que se ordenaban las parcelas fueron analizados mediante un Análisis de Correspondencia Destendenciado (DCA) realizado con la función decorana del paquete vegan
(Oksanen et al., 2019). Con el mismo paquete y la función hclust se realizó la clasificación de las parcelas en función de su vegetación mediante una clasificación jerárquica aglomerativa, utilizando la distancia de Bray-Curtis y el ligamiento de Ward. Se aplicaron dos niveles de corte considerando los 30 procedimientos provistos en el paquete $\mathrm{NbClust}$ (Charrad et al., 2014). Las unidades de vegetación correspondieron a los grupos formados por el nivel de corte más frecuente de todos los considerados. Las comunidades de plantas fueron establecidas mediante el nivel de corte sugerido por los métodos Duda y Pseudot2 (Duda \& Hart, 1973) debido a que han demostrado mejores desempeños en comparación con otros índices según Milligan \& Cooper (1985) y Chouikhi et al. (2015). Debido a que algunas de las parcelas presentaron el césped cortado, sólo aquellas gramíneas que por sus características vegetativas y ecológicas resultaron inconfundibles (Arundo donax, Cortaderia selloana, Echinochloa polystachya y Louisiella elephantipes) se trataron independientemente en los análisis multivariados, mientras que las restantes fueron tratadas en conjunto dentro de la categoría "Poaceae spp.", dando como resultado una matriz de 140 taxones por 77 parcelas.

\section{RESULTADOS}

\section{Riqueza de plantas vasculares de la RECU-CN}

En el relevamiento se registraron 154 taxones correspondientes a un total de 144 especies, 123 géneros y 58 familias de plantas vasculares. Las especies Ligustrum sinense, Ligustrum lucidum y Melia azedarach resultaron ser las más abundantes y frecuentes, y las familias Poaceae y Asteraceae, las que tuvieron más representantes. Se registraron 79 especies nativas de la región (58\% del total de las especies determinadas), que representaron cerca del $30 \%$ de la cobertura total. La riqueza total estimada de la RECU-CN fue de 238 especies con un intervalo de confianza del $95 \%$ de entre 195 y 327 especies. En cuanto a las formas de vida se registraron árboles, arbustos, subarbustos, hierbas y trepadoras, siendo las de mayor cobertura los árboles (46\%) y las hierbas (44\%) mientras que los arbustos, subarbustos y trepadoras se encontraron en baja proporción (3\%, 3\% y 4\% respectivamente). 
Tabla 1. Listado de especies registradas en la Reserva Ecológica Ciudad Universitaria - Costanera Norte. Se indica si la especie es nativa en la región, su forma de vida, frecuencia y cobertura en cada comunidad por unidad de vegetación. Abreviaturas: H, Humedal; B, Bosque; P, Pastizal con arboledas; comunidades: 1, Canutillar de Louisiella elephantipes; 2, Verdolagal de Ludwigia sp.; 3, Bosque de ceibo y ligustrina; 4, Bosque de ligustro y paraíso; 5, Bosque de eucalipto y ligustrina; 6, Cañaveral de caña de Castilla; 7, Arboleda de tipa; 8, Pastizal con ricino; 9, Pastizal con carda; 10, Pastizal mixto; E, exótica; N, nativa; ND, no determinada; $\mathbf{F V}$, forma de vida; $\mathbf{A}$, árbol; $\mathbf{B}$, arbusto; $\mathbf{H}$, hierba; $\mathbf{S}$, subarbusto; $\mathbf{T}$, trepadora; frecuencias: a, 1-20\%; b, 21-40\%; c, 41-60\%; d, 61-80\%; e, 81-100\%; coberturas: 1, 0-0,1\%; 2, 0,1-1\%; 3, $1-5 \% ; \mathbf{4}, 5-10 \% ; \mathbf{5}, 10-25 \% ; \mathbf{6}, 25-33 \% ; \mathbf{7}, 33-50 \% ; \mathbf{8}, 50-75 \%$ y $\mathbf{9}, 75-100 \%$.

\begin{tabular}{|c|c|c|c|c|c|c|c|c|c|c|c|}
\hline \multirow{3}{*}{ Taxones } & \multirow{2}{*}{\multicolumn{2}{|c|}{$\begin{array}{c}\text { Unidad de vegetación } \\
\text { Comunidad }\end{array}$}} & \multicolumn{2}{|c|}{$\mathbf{H}$} & \multicolumn{3}{|c|}{$\mathbf{B}$} & \multicolumn{4}{|c|}{$\mathbf{P}$} \\
\hline & & & \multirow[t]{2}{*}{1} & \multirow[t]{2}{*}{2} & \multirow[t]{2}{*}{3} & \multirow[t]{2}{*}{4} & \multirow{2}{*}{5} & \multirow[t]{2}{*}{7} & \multirow[t]{2}{*}{8} & \multirow[t]{2}{*}{9} & \multirow[t]{2}{*}{10} \\
\hline & Origen & FV & & & & & & & & & \\
\hline Alismataceae & & & & & & & & & & & \\
\hline Echinodorus grandiflorus (Cham. \& Schltdl.) Micheli & $\mathrm{N}$ & $\mathrm{H}$ & & $\mathrm{d} 4$ & & & a1 & & & & \\
\hline Sagittaria montevidensis Cham. \& Schltdl. & $\mathrm{N}$ & $\mathrm{H}$ & & $\mathrm{a} 2$ & & & & & & & \\
\hline Amaryllidaceae & & & & & & & & & & & \\
\hline Zephyranthes candida (Herb. ex Lindl.) Herb. & $\mathrm{N}$ & $\mathrm{H}$ & & & a3 & $\mathrm{a} 2$ & & & & & \\
\hline Apiaceae & & & & & & & & & & & \\
\hline Conium maculatum $\mathrm{L}$. & $\mathrm{E}$ & $\mathrm{H}$ & & & & & a1 & & c3 & a1 & a1 \\
\hline Daucus pusillus Michx. & $\mathrm{N}$ & $\mathrm{H}$ & & & & & & $\mathrm{b} 2$ & & & b3 \\
\hline Eryngium pandanifolium Cham. \& Schltdl. & $\mathrm{N}$ & $\mathrm{H}$ & & $\mathrm{c} 4$ & b3 & & b2 & & & & \\
\hline Hydrocotyle sp. & $\mathrm{N}$ & $\mathrm{H}$ & & & $\mathrm{d} 3$ & a2 & b3 & & & & \\
\hline Lilaeopsis brasiliensis (Glaz.) Affolter & $\mathrm{N}$ & $\mathrm{H}$ & & a1 & & & & & & & \\
\hline Apocynaceae & & & & & & & & & & & \\
\hline Orthosia virgata (Poir.) E. Fourn. & $\mathrm{N}$ & $\mathrm{T}$ & & & $\mathrm{a} 2$ & $\mathrm{a} 2$ & $\mathrm{a} 2$ & & & & \\
\hline Araceae & & & & & & & & & & & \\
\hline Alocasia macrorrhizos (L.) G. Don & $\mathrm{E}$ & $\mathrm{H}$ & & & a2 & & & & & & \\
\hline Arum italicum Miller & $\mathrm{E}$ & $\mathrm{H}$ & & & $\mathrm{b} 2$ & a2 & & & & & \\
\hline Pistia stratiotes $\mathrm{L}$. & $\mathrm{N}$ & $\mathrm{H}$ & & a1 & & & & & & & \\
\hline Araliaceae & & & & & & & & & & & \\
\hline Hedera helix $\mathrm{L}$. & $\mathrm{E}$ & $\mathrm{T}$ & & & $\mathrm{c} 5$ & a4 & & & & & \\
\hline Arecaceae & & & & & & & & & & & \\
\hline Phoenix canariensis Chabaud & $\mathrm{E}$ & A & & & a1 & a3 & & & & & \\
\hline Asparagaceae & & & & & & & & & & & \\
\hline Asparagus densiflorus (Kunth) Jessop & $\mathrm{E}$ & $\mathrm{H}$ & & & & a1 & & & & & \\
\hline Asteraceae & & & & & & & & & & & \\
\hline Artemisia aппиа $\mathrm{L}$. & $\mathrm{E}$ & $\mathrm{H}$ & & & & & & a1 & & $\mathrm{d} 3$ & $\mathrm{~b} 2$ \\
\hline Baccharis salicifolia (Ruiz \& Pav.) Pers. & $\mathrm{N}$ & $\mathrm{B}$ & & & & a2 & a1 & a2 & $\mathrm{c} 3$ & d3 & b2 \\
\hline Bidens pilosa $\mathrm{L}$. & $\mathrm{N}$ & $\mathrm{H}$ & & & & & a1 & & c3 & $\mathrm{a} 2$ & a1 \\
\hline Calyptocarpus biaristatus (DC.) H. Rob. & $\mathrm{N}$ & $\mathrm{H}$ & & & b3 & $\mathrm{a} 2$ & a1 & & & & \\
\hline Cirsium vulgare (Savi) Ten. & $\mathrm{E}$ & $\mathrm{H}$ & & & & & & & b2 & a2 & $\mathrm{c} 2$ \\
\hline Conyza bonariensis (L.) Cronquist & $\mathrm{N}$ & $\mathrm{H}$ & & & & & a1 & & & a2 & a1 \\
\hline Cotula australis (Sieber ex Spreng.) Hook. f. & $\mathrm{E}$ & $\mathrm{H}$ & & & & & & & & a1 & a2 \\
\hline Gamochaeta pensylvanica (Willd.) Cabrera & $\mathrm{N}$ & $\mathrm{H}$ & & & & & & & & a1 & $\mathrm{b} 2$ \\
\hline Gamochaeta sp. & $\mathrm{N}$ & $\mathrm{H}$ & & & & & & & & & a1 \\
\hline Gymnocoronis spilanthoides (D. Don ex Hook. \& Arn.) DC. & $\mathrm{N}$ & $\mathrm{H}$ & a3 & a1 & & & & & & & \\
\hline Helminthotheca echioides (L.) Holub & $\mathrm{E}$ & $\mathrm{H}$ & & & & & & & & c3 & a2 \\
\hline Lactuca serriola $\mathrm{L}$. & $\mathrm{E}$ & $\mathrm{H}$ & & & & & & & & & a1 \\
\hline Senecio madagascariensis Poir. & $\mathrm{E}$ & $\mathrm{H}$ & & & & & & & & & a2 \\
\hline Silybum marianum (L.) Gaertn. & $\mathrm{E}$ & $\mathrm{H}$ & & & & & & & b2 & b3 & $\mathrm{c} 2$ \\
\hline Solidago chilensis Meyen & $\mathrm{N}$ & $\mathrm{H}$ & & & & & & & & a2 & a1 \\
\hline Symphyotrichum squamatum (Spreng.) G.L. Nesom & $\mathrm{N}$ & $\mathrm{H}$ & & & & & & & & $\mathrm{a} 2$ & \\
\hline Taraxacum officinale F.H. Wigg. & $\mathrm{E}$ & $\mathrm{H}$ & & & & & & a1 & & b2 & a1 \\
\hline Bignoniaceae & & & & & & & & & & & \\
\hline Dolichandra unguis-cati (L.) L.G. Lohmann & $\mathrm{N}$ & $\mathrm{T}$ & & & & b3 & & & & & \\
\hline
\end{tabular}


Tabla 1. (Continuación).

\begin{tabular}{|c|c|c|c|c|c|c|c|c|c|c|c|c|}
\hline \multirow{3}{*}{ Taxones } & \multirow{2}{*}{\multicolumn{2}{|c|}{$\begin{array}{c}\text { Unidad de vegetación } \\
\text { Comunidad }\end{array}$}} & \multicolumn{2}{|c|}{$\mathbf{H}$} & \multicolumn{4}{|c|}{ B } & \multicolumn{4}{|c|}{$\mathbf{P}$} \\
\hline & & & \multirow[t]{2}{*}{1} & \multirow[t]{2}{*}{2} & \multirow[t]{2}{*}{3} & \multirow[t]{2}{*}{4} & \multirow[t]{2}{*}{5} & \multirow[t]{2}{*}{6} & \multirow[t]{2}{*}{7} & \multirow[t]{2}{*}{8} & \multirow[t]{2}{*}{9} & \multirow[t]{2}{*}{10} \\
\hline & Origen & FV & & & & & & & & & & \\
\hline Boraginaceae & & & & & & & & & & & & \\
\hline Echium plantagineum $\mathrm{L}$. & $\mathrm{E}$ & $\mathrm{H}$ & & & & & & & & & a1 & \\
\hline Brassicaceae & & & & & & & & & & & & \\
\hline Hirschfeldia incana (L.) Lagr.-Foss. & $\mathrm{E}$ & $\mathrm{H}$ & & & & & & & a3 & b1 & & b2 \\
\hline Bromeliaceae & & & & & & & & & & & & \\
\hline Tillandsia recurvata (L.) L. & $\mathrm{N}$ & $\mathrm{H}$ & & & & a1 & & & & & & \\
\hline Cannabaceae & & & & & & & & & & & & \\
\hline Celtis australis $\mathrm{L}$. & $\mathrm{E}$ & A & & & & & & & a3 & & & \\
\hline Celtis tala Gillies ex Planch. & $\mathrm{N}$ & A & & & & & & & & b4 & & \\
\hline Caprifoliaceae & & & & & & & & & & & & \\
\hline Dipsacus fullonum L. & $\mathrm{E}$ & $\mathrm{H}$ & & & & & a3 & & $\mathrm{c} 5$ & $\mathrm{~d} 5$ & e5 & b4 \\
\hline Caryophyllaceae & & & & & & & & & & & & \\
\hline Stellaria media (L.) Cirillo & $\mathrm{E}$ & $\mathrm{H}$ & & & & & & & & & a1 & \\
\hline Chenopodiaceae & & & & & & & & & & & & \\
\hline Dysphania ambrosioides (L.) Mosyakin \& Clemants & $\mathrm{N}$ & $\mathrm{H}$ & & & & & & & & & & a1 \\
\hline Commelinaceae & & & & & & & & & & & & \\
\hline Tradescantia fluminensis Vell. & $\mathrm{N}$ & $\mathrm{H}$ & a3 & $\mathrm{a} 2$ & $\mathrm{c} 5$ & & a2 & & & & & \\
\hline Tripogandra diuretica (Mart.) Handlos & $\mathrm{N}$ & $\mathrm{H}$ & & & e5 & a1 & a3 & & & & & \\
\hline Convolvulaceae & & & & & & & & & & & & \\
\hline Convolvulus arvensis $\mathrm{L}$. & $\mathrm{E}$ & $\mathrm{T}$ & & & & & & & & b1 & & \\
\hline Dichondra microcalyx (Hallier f.) Fabris & $\mathrm{N}$ & $\mathrm{H}$ & & & a4 & & b3 & & a1 & & & b3 \\
\hline Ipomoea alba $\mathrm{L}$. & $\mathrm{N}$ & $\mathrm{T}$ & & $\mathrm{b} 2$ & & $\mathrm{a} 2$ & & & & & & \\
\hline Ipomoea cairica $(\mathrm{L}$.$) Sweet$ & $\mathrm{N}$ & $\mathrm{T}$ & & a1 & a1 & b2 & a2 & & a2 & $\mathrm{b} 2$ & $\mathrm{a} 2$ & \\
\hline Ipomoea indica (Burm.) Merr. & $\mathrm{N}$ & $\mathrm{T}$ & & & & a1 & & $\mathrm{c} 1$ & & b3 & & \\
\hline Cucurbitaceae & & & & & & & & & & & & \\
\hline Cayaponia bonariensis (Mill.) Mart.Crov. & $\mathrm{N}$ & $\mathrm{T}$ & & & & a2 & & & & & & \\
\hline Cyperaceae & & & & & & & & & & & & \\
\hline Carex sp. & ND & $\mathrm{H}$ & & & $\mathrm{b} 2$ & a1 & b3 & & & & & \\
\hline Cyperaceae sp. & ND & $\mathrm{H}$ & & a1 & & & & & & & & \\
\hline Cyperus eragrostis Lam. & $\mathrm{N}$ & $\mathrm{H}$ & $\mathrm{a} 2$ & b1 & $\mathrm{a} 2$ & & & & & & & a2 \\
\hline Schoenoplectus californicus (C.A. Mey.) Soják & $\mathrm{N}$ & $\mathrm{H}$ & & a3 & & & & & & & & \\
\hline Euphorbiaceae & & & & & & & & & & & & \\
\hline Euphorbia peplus L. & $\mathrm{E}$ & $\mathrm{H}$ & & & & a2 & & & & & & \\
\hline Euphorbia serpens Kunth & $\mathrm{N}$ & $\mathrm{H}$ & & & & & & & & & & b2 \\
\hline Manihot grahamii Hook. & $\mathrm{N}$ & A & & & & $\mathrm{a} 2$ & a1 & & & & & \\
\hline Ricinus communis $\mathrm{L}$. & $\mathrm{E}$ & $\mathrm{S}$ & & & & a2 & & $\mathrm{c} 1$ & b3 & $\mathrm{e} 5$ & b3 & \\
\hline Sapium haematospermum Müll. Arg. & $\mathrm{N}$ & $\mathrm{A}$ & & & & a3 & a3 & & & & a1 & \\
\hline Fabaceae & & & & & & & & & & & & \\
\hline Canavalia bonariensis Lindl. & $\mathrm{N}$ & $\mathrm{T}$ & & & a2 & & & & & & & \\
\hline Erythrina crista-galli $\mathrm{L}$. & $\mathrm{N}$ & A & & & e5 & a3 & & & & & & \\
\hline Fabaceae sp. & ND & $\mathrm{T}$ & & $\mathrm{a} 2$ & & & & & & & & \\
\hline Galega officinalis L. & $\mathrm{E}$ & $\mathrm{H}$ & & & & & & & a2 & & a1 & a3 \\
\hline Lotus tenuis Waldst. \& Kit. ex Willd. & $\mathrm{E}$ & $\mathrm{H}$ & & & & & & & a2 & $\mathrm{b} 2$ & & b3 \\
\hline Medicago lupulina $\mathrm{L}$. & $\mathrm{E}$ & $\mathrm{H}$ & & & & & & & & & & a3 \\
\hline Melilotus albus Desr. & $\mathrm{E}$ & $\mathrm{S}$ & & & & & & & & & e4 & \\
\hline Robinia pseudoacacia L. & $\mathrm{E}$ & A & & & & & a3 & & & & & \\
\hline Tipuana tipu (Benth.) Kuntze & $\mathrm{E}$ & $\mathrm{A}$ & & & & & b5 & & e8 & & & a2 \\
\hline Trifolium repens $\mathrm{L}$. & $\mathrm{E}$ & $\mathrm{H}$ & & & & & & & a2 & & & e4 \\
\hline Vachellia caven (Molina) Seigler \& Ebinger & $\mathrm{N}$ & A & & & & & & & & & $\mathrm{a} 2$ & \\
\hline Vicia sativa $\mathrm{L}$ & $\mathrm{E}$ & $\mathrm{H}$ & & & & & & & $\mathrm{a} 2$ & $\mathrm{c} 3$ & c3 & $\mathrm{c} 2$ \\
\hline
\end{tabular}


Tabla 1. (Continuación).

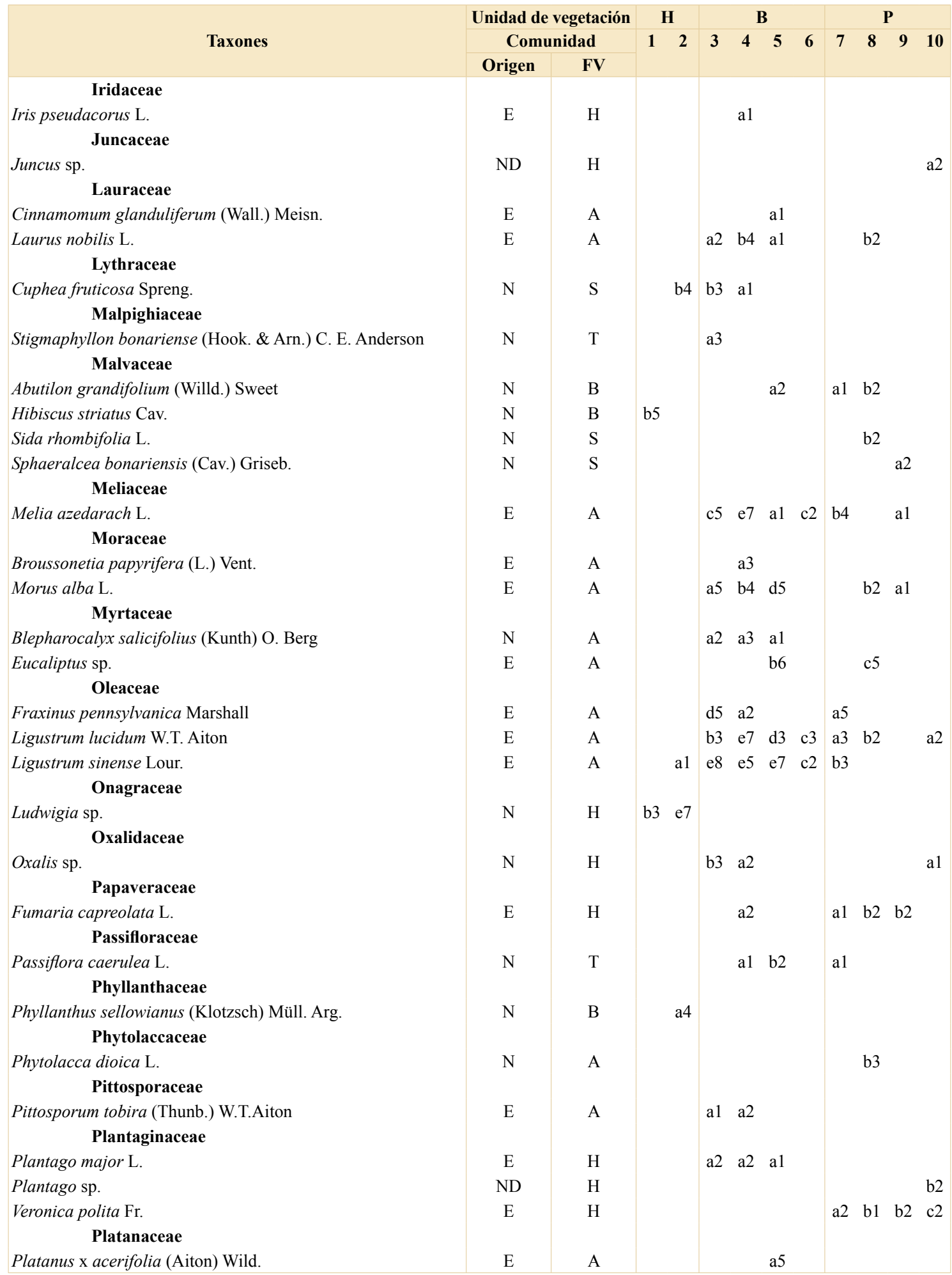


Tabla 1. (Continuación).

\begin{tabular}{|c|c|c|c|c|c|c|c|c|c|c|c|c|}
\hline \multirow{3}{*}{ Taxones } & \multirow{2}{*}{\multicolumn{2}{|c|}{$\begin{array}{c}\text { Unidad de vegetación } \\
\text { Comunidad }\end{array}$}} & \multicolumn{2}{|c|}{$\mathbf{H}$} & \multicolumn{4}{|c|}{ B } & \multicolumn{4}{|c|}{$\mathbf{P}$} \\
\hline & & & \multirow[t]{2}{*}{1} & \multirow[t]{2}{*}{2} & \multirow[t]{2}{*}{3} & \multirow[t]{2}{*}{4} & \multirow[t]{2}{*}{5} & \multirow[t]{2}{*}{6} & \multirow[t]{2}{*}{7} & \multirow[t]{2}{*}{8} & \multirow[t]{2}{*}{9} & \multirow[t]{2}{*}{10} \\
\hline & Origen & FV & & & & & & & & & & \\
\hline Poaceae & & & & & & & & & & & & \\
\hline Arundo donax L. & $\mathrm{E}$ & $\mathrm{H}$ & & & & a3 & & e9 & & b3 & a3 & \\
\hline Cortaderia selloana (Schult. \& Schult. f.) Asch. \& Graebn. & $\mathrm{N}$ & $\mathrm{H}$ & & & & & a2 & & & & a2 & \\
\hline Echinochloa polystachya (Kunth) Hitchc. & $\mathrm{N}$ & $\mathrm{H}$ & a5 & a3 & & & & & & & & \\
\hline Louisiella elephantipes (Nees ex Trin.) Zuloaga & $\mathrm{N}$ & $\mathrm{H}$ & e7 & a1 & & $\mathrm{a} 2$ & & & & & & \\
\hline Poaceae spp. ${ }^{\mathrm{a}}$ & ND & $\mathrm{H}$ & & & & b5 & b3 & & d5 & e6 & e6 & e5 \\
\hline Polygonaceae & & & & & & & & & & & & \\
\hline Polygonum sp.1 & ND & $\mathrm{H}$ & b3 & b4 & a1 & a2 & & & & & & \\
\hline Polygonum sp.2 & ND & $\mathrm{H}$ & & a3 & & & & & & & & \\
\hline Polygonum sp.3 & ND & $\mathrm{H}$ & & & a3 & & a2 & & & & & \\
\hline Polygonum sp.4 & ND & $\mathrm{H}$ & $\mathrm{a} 2$ & d3 & a1 & & & & & & & \\
\hline Polygonum sp.5 & ND & $\mathrm{H}$ & a3 & a2 & & & & & & & & \\
\hline Rumex acetosella $\mathrm{L}$. & $\mathrm{E}$ & $\mathrm{H}$ & & & & & & & & & & $\mathrm{a} 2$ \\
\hline Pontederiaceae & & & & & & & & & & & & \\
\hline Pontederia crassipes Mart. & $\mathrm{N}$ & $\mathrm{H}$ & & a1 & & & & & & & & \\
\hline Pontederia sp. & $\mathrm{N}$ & $\mathrm{H}$ & a2 & & & & & & & & & \\
\hline Portulacaceae & & & & & & & & & & & & \\
\hline Portulaca sp. & ND & $\mathrm{H}$ & & & & & & & & & & a2 \\
\hline Primulaceae & & & & & & & & & & & & \\
\hline Anagallis arvensis $\mathrm{L}$. & $\mathrm{E}$ & $\mathrm{H}$ & & & & & & & & & & $\mathrm{a} 2$ \\
\hline Pteridaceae & & & & & & & & & & & & \\
\hline Adiantum raddianum C. Presl & $\mathrm{N}$ & $\mathrm{H}$ & & & & a1 & a1 & & & & & \\
\hline Pteris vittata $\mathrm{L}$. & $\mathrm{N}$ & $\mathrm{H}$ & & & & a2 & a1 & & & & & \\
\hline Ranunculaceae & & & & & & & & & & & & \\
\hline Ranunculus apiifolius Pers. & $\mathrm{N}$ & $\mathrm{H}$ & b2 & & & & & & & & & \\
\hline Rubiaceae & & & & & & & & & & & & \\
\hline Galianthe brasiliensis (Spreng.) E.L. Cabral \& Bacigalupo & $\mathrm{N}$ & $\mathrm{S}$ & & & a1 & & & & & & & \\
\hline Galium aparine $\mathrm{L}$. & $\mathrm{E}$ & $\mathrm{H}$ & & & & c2 & & & $\mathrm{c} 4$ & $\mathrm{~d} 3$ & $\mathrm{a} 2$ & b2 \\
\hline Salicaceae & & & & & & & & & & & & \\
\hline Salix humboldtiana Willd. & $\mathrm{N}$ & A & & b4 & & & a2 & & & & & \\
\hline Salix sp. & $\mathrm{E}$ & A & & & & & a3 & & & & & \\
\hline Sapindaceae & & & & & & & & & & & & \\
\hline Acer negundo L. & $\mathrm{E}$ & A & & a1 & a3 & b3 & $\mathrm{d} 3$ & & $\mathrm{c} 3$ & b2 & & \\
\hline Simaroubaceae & & & & & & & & & & & & \\
\hline Ailanthus altissima (Mill.) Swingle & $\mathrm{E}$ & A & & & & & & & a3 & & & \\
\hline Solanaceae & & & & & & & & & & & & \\
\hline Jaborosa integrifolia Lam. & $\mathrm{N}$ & $\mathrm{H}$ & & & & & & & & & & a3 \\
\hline Physalis viscosa $\mathrm{L}$. & $\mathrm{N}$ & $\mathrm{B}$ & & & & & & & & b2 & & \\
\hline Salpichroa origanifolia (Lam.) Baill. & $\mathrm{N}$ & S & & & & & & & & b2 & & \\
\hline Solanum amygdalifolium Steud. & $\mathrm{N}$ & $\mathrm{B}$ & & a2 & & & & & & & & \\
\hline Solanum bonariense L. & $\mathrm{N}$ & $\mathrm{B}$ & & & & & & & & b2 & & \\
\hline Solanum pilcomayense Morong & $\mathrm{N}$ & $\mathrm{S}$ & & & & & & & a2 & & & \\
\hline Solanum sisymbriifolium Lam. & $\mathrm{N}$ & $\mathrm{S}$ & & & & & & & a1 & & & \\
\hline Thelypteridaceae & & & & & & & & & & & & \\
\hline aff. Goniopteris sp. & ND & $\mathrm{H}$ & & & b3 & $\mathrm{a} 2$ & a1 & & & & & \\
\hline
\end{tabular}

a Dentro de Poaceae spp. se identificaron las especies nativas Bromus catharticus Vahl, Paspalum notatum Flüggé y Paspalum urvillei Steud. y las exóticas Cynodon dactylon (L.) Pers., Lolium sp., Megathyrsus maximus (Jacq.) B.K. Simon \& S.W.L. Jacobs, Poa annua L., Polypogon sp., Setaria sp., Sorghum halepense (L.) Pers. Otros cinco morfotipos de gramíneas sólo se identificaron a nivel de familia. 
Tabla 1. (Continuación).

\begin{tabular}{|c|c|c|c|c|c|c|c|c|c|c|c|c|}
\hline \multirow{3}{*}{ Taxones } & \multirow{2}{*}{\multicolumn{2}{|c|}{$\begin{array}{l}\text { Unidad de vegetación } \\
\text { Comunidad }\end{array}$}} & \multicolumn{2}{|c|}{$\mathbf{H}$} & \multicolumn{4}{|c|}{ B } & \multicolumn{4}{|c|}{$\mathbf{P}$} \\
\hline & & & \multirow[t]{2}{*}{1} & \multirow[t]{2}{*}{2} & \multirow[t]{2}{*}{3} & \multirow[t]{2}{*}{4} & \multirow[t]{2}{*}{5} & \multirow[t]{2}{*}{6} & \multirow[t]{2}{*}{7} & \multirow[t]{2}{*}{8} & \multirow{2}{*}{\multicolumn{2}{|c|}{9}} \\
\hline & Origen & FV & & & & & & & & & & \\
\hline Urticaceae & & & & & & & & & & & & \\
\hline Parietaria debilis G. Forst. & $\mathrm{N}$ & $\mathrm{H}$ & & & & a1 & a2 & & & & & a1 \\
\hline Parietaria judaica L. & $\mathrm{E}$ & $\mathrm{H}$ & & & & a1 & b4 & & & b3 & & \\
\hline Verbenaceae & & & & & & & & & & & & \\
\hline Lantana camara $\mathrm{L}$. & $\mathrm{N}$ & B & & & & a1 & & & & & & \\
\hline Verbena aff. bonariensis L. & $\mathrm{N}$ & $\mathrm{H}$ & & & & & a1 & & $\mathrm{a} 2$ & $\mathrm{c} 3$ & b2 & b3 \\
\hline Vitaceae & & & & & & & & & & & & \\
\hline Cissus palmata Poir. & $\mathrm{N}$ & $\mathrm{T}$ & & & a1 & & & & & & & \\
\hline Cissus verticillata (L.) Nicolson \& C.E. Jarvis & $\mathrm{N}$ & $\mathrm{T}$ & & a2 & & a2 & b4 & & & & & \\
\hline Eudicotiledóneas & & & & & & & & & & & & \\
\hline Eudicotiledónea sp.1 & ND & $\mathrm{H}$ & & & & & & & & & a1 & \\
\hline Eudicotiledónea sp.2 & ND & $\mathrm{H}$ & & & & & & & & & & a1 \\
\hline Eudicotiledónea sp.3 & ND & $\mathrm{H}$ & & b2 & & & & & & & & \\
\hline
\end{tabular}

\section{Unidades de vegetación y comunidades de la RECU-CN}

Los ejes DCA1 y DCA2 tuvieron autovalores de 0,9 y 0,4 y explicaron el $6 \%$ y el $3 \%$ de la variabilidad de los datos respectivamente (Fig. 2). En el extremo positivo del eje DCA1 se observó un conglomerado de parcelas que pertenecen a la porción anegada del área de estudio, ubicadas entre el murallón de contención de Ciudad Universitaria y la porción elevada del terreno que corresponde a la península de relleno y, también, sobre la porción inundable costera del área. En el sector central del ordenamiento se encontraron las parcelas ubicadas sobre la península, desde la orilla inundable hasta el centro de la misma, donde el terreno pasa a ser más elevado y disminuye la posibilidad de ingreso de agua ante eventos extraordinarios de sudestada. En el extremo negativo del eje DCA1 se agruparon las parcelas correspondientes a la porción de la península más cercana al río, cuyos rellenos son más elevados que en la porción antedicha, y se encuentran completamente aislados de la dinámica estuarial, siendo a su vez la porción más antropizada. De esta forma se identificó en forma indirecta un gradiente ambiental de inundación hacia la derecha de la figura y, en sentido opuesto al anterior, un gradiente de antropización que incluye tanto la existencia de un sustrato antrópico, como la de disturbios producidos por el humano (Fig. 2).

El nivel de corte más frecuente de todos los considerados en el análisis de clasificación indicó la formación de tres grandes grupos de parcelas (distintos trazos en la figura 2). Con base en esta clasificación se definieron las unidades de vegetación "Humedal", "Bosque" y "Pastizal con arboledas", con 15, 29 y 33 parcelas respectivamente. El segundo nivel de corte indicó la formación de 10 grupos (Fig. 2) que definieron las 10 comunidades de plantas de entre dos y 16 parcelas cada uno. La formación de estos 10 grupos de parcelas se dio en un contexto de variabilidad explicada de los ejes del ordenamiento cercana al $10 \%$ y son gráficamente menos evidentes que los tres grupos correspondientes a las unidades de vegetación (Fig. 2). Tanto las unidades de vegetación como las comunidades fueron denominadas en función de sus formas de vida y especies dominantes y se describen a continuación.

Humedal. Esta unidad se ubicó principalmente entre el murallón de contención de Ciudad Universitaria y la península, con una parcela sobre la costa (Fig. 1). Esta unidad de vegetación tuvo el menor número de especies totales (32) con un promedio de cinco especies por parcela. Por otro lado, tanto el porcentaje de número de especies nativas $(90 \%)$ como el de cobertura de dichas especies $(99 \%)$ resultaron ser mayores que en las otras unidades. Esta unidad de vegetación presentó mayor cobertura de especies herbáceas (77\%), siendo las más abundantes y frecuentes Ludwigia sp. y Louisiella elephantipes (Fig. 3). 
Otras especies de herbáceas registradas con coberturas y frecuencias intermedias resultaron ser distintas especies de Polygonum, Echinodorus grandiflorus, Echinochloa polystachya y Eryngium pandanifolium; también se registraron especies arbustivas y arbóreas como Hibiscus striatus y Salix humboldtiana respectivamente (Fig. 3).
Con una cobertura intermedia, pero una baja frecuencia se registró Schoenoplectus californicus, mientras que por el contrario la trepadora Ipomoea alba y la herbácea Cyperus eragrostis se registraron en una frecuencia intermedia, pero con baja cobertura (Tabla 1). En esta unidad se identificaron las comunidades (1)Canutillar de Louisiella elephantipes

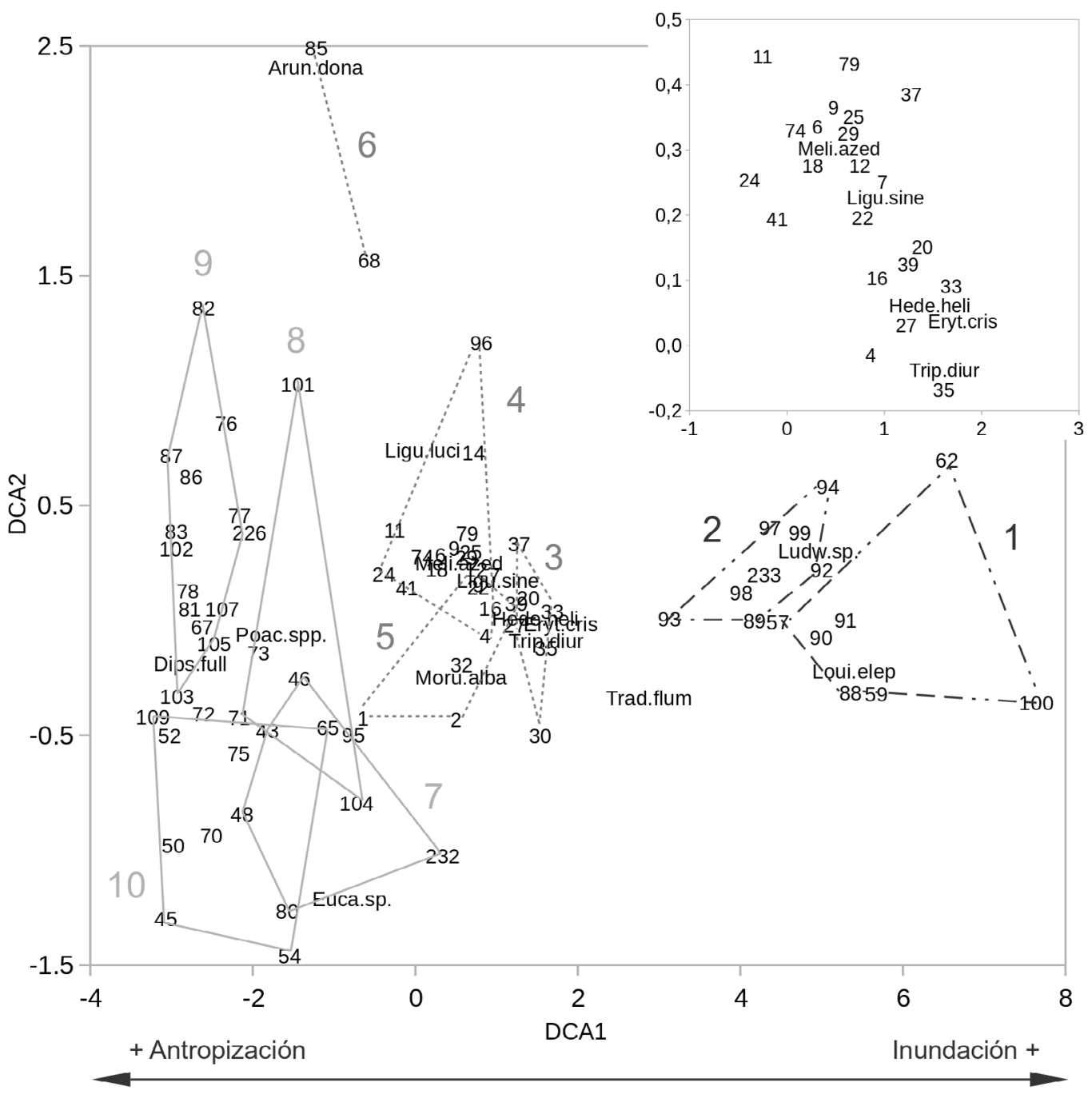

Fig. 2. Ordenamiento y clasificación de la vegetación de la RECU-CN. En el ordenamiento se indican las 15 especies de mayor cobertura (letras), las parcelas de muestreo (números pequeños), una ampliación de la zona central congestionada (esquina superior derecha) y el gradiente ambiental identificado (flecha abajo). En la clasificación se indican los grupos de parcelas correspondientes a las tres unidades de vegetación (Humedal, líneas de rayas y puntos; Bosque, líneas punteadas; Pastizal con arboledas, líneas continuas) y a las 10 comunidades vegetales (números grandes, ver figura 1). Arun.dona $=$ Arundo donax, Dips.full $=$ Dipsacus fullonum, Eryt.cris $=$ Erythrina crista-galli, Euca.sp. $=$ Eucalyptus sp., Hede.heli = Hedera helix, Ligu.luci $=$ Ligustrum lucidum, Ligu.sine $=$ Ligustrum sinense, Loui.elep $=$ Louisiella elephantipes, Ludw.sp. = Ludwigia sp., Meli.azed = Melia azedarach, Moru.alba = Morus alba, Poac.spp. $=$ Poaceae spp., Tipu.tipu $=$ Tipuana tipu, Trad.flum $=$ Tradescantia fluminensis, Trip.diur $=$ Tripogranda diuretica. 
y (2) Verdolagal de Ludwigia sp. con un total de 7 y 8 parcelas respectivamente. La composición de especies de cada comunidad se detalla en la Tabla 1.

Bosque. Esta segunda unidad de vegetación se ubicó en la porción central del área (Fig. 1). Se registraron 77 especies con un promedio de 10 especies por parcela, de las cuales el 55\% correspondieron a especies nativas de la región con una cobertura total del $18 \%$. En promedio las parcelas se encontraron cubiertas en un $72 \%$ por árboles. Las especies que se encontraron con mayor cobertura y frecuencia fueron Ligustrum sinense, Ligustrum lucidum y Melia azedarach, dominando sobre otras especies arbóreas como Morus alba, Erythrina crista-galli y Acer negundo (Fig. 3). Además, se registraron hierbas como Tripogandra diuretica y gramíneas (Poaceae spp.). En esta unidad se identificaron las comunidades (3) Bosque de ceibo y ligustrina, (4) Bosque de ligustro y paraíso, (5) Bosque de eucalipto y ligustrina y (6) Cañaveral de caña de Castilla, con un total de 5, 16, 6 y 2 parcelas respectivamente. La composición de especies de cada comunidad se detalla en la Tabla 1.

Pastizal con arboledas. Esta tercera unidad de vegetación se ubicó en la península hacia el río, ocupando la superficie ovalada rodeada por cemento y las zonas lindantes que limitan con la franja boscosa (Fig. 1). Se registraron 77 especies en total y aproximadamente 9 por parcela. Esta unidad de vegetación presentó el menor porcentaje de número de especies nativas de las tres (43\%) y de cobertura total correspondiente a este grupo de especies (11\%). La forma de vida predominante, con un $66 \%$ de cobertura, correspondió a las herbáceas, quedando el restante $34 \%$ mayormente comprendido por árboles. Esta unidad de vegetación se encontró dominada, tanto en cobertura como en frecuencia, por diversas especies de gramíneas (Poaceae spp.), como Megathyrsus maximus y Sorghum halepense, acompañadas principalmente por Dipsacus fullonum (Fig. 3). Otras herbáceas fueron registradas en una frecuencia intermedia, pero con coberturas menores, como por ejemplo Melilotus albus, Vicia sativa y Trifolium repens. En cuanto a otras formas de vida se registraron considerables cobertura y frecuencia de la especie arbórea Tipuana tipu y los arbustos Baccharis salicifolia y Ricinus communis. En esta unidad se identificaron las comunidades (7) Arboleda de tipa, (8) Pastizal con ricino, (9) Pastizal con carda y (10) Pastizal mixto, con un total de 6, 4, 14 y 9 parcelas respectivamente. La composición de especies de cada comunidad se detalla en la Tabla 1.

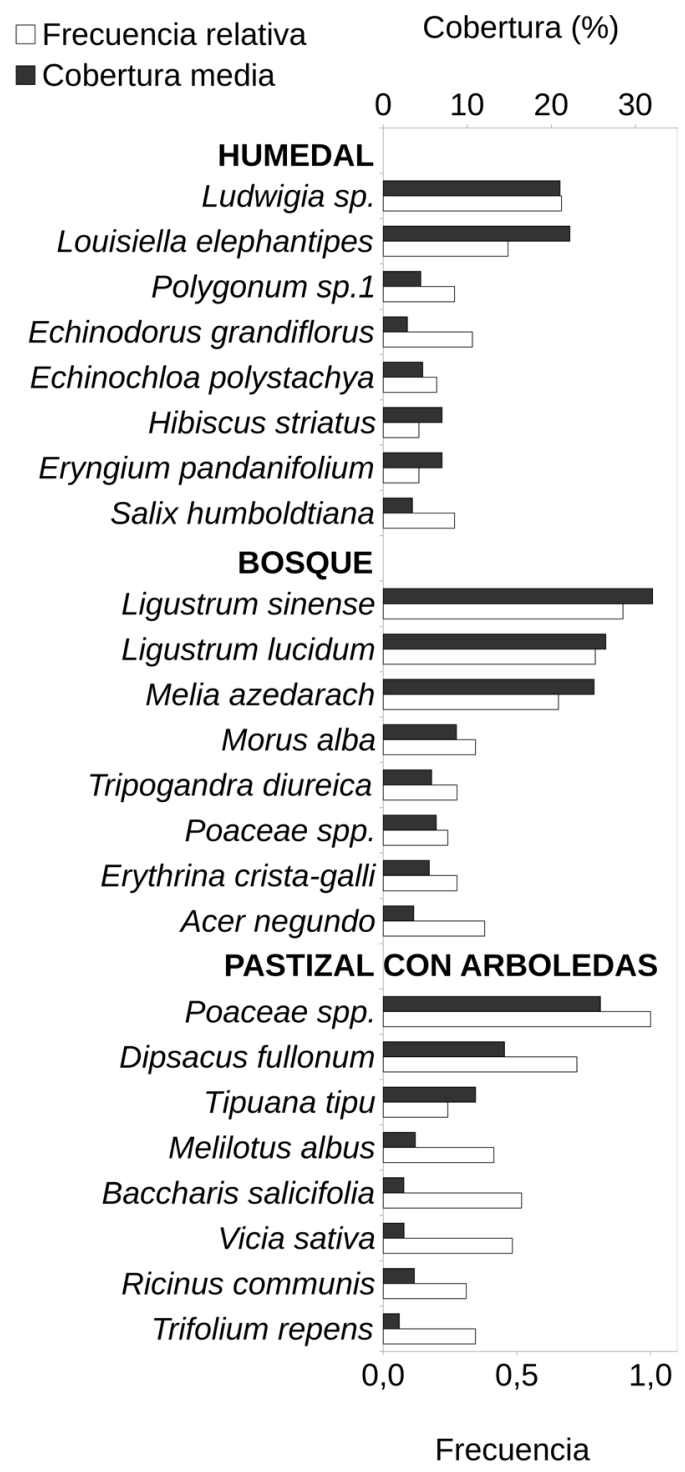

Fig. 3. Cobertura media (barras negras) y frecuencia relativa (barras blancas) de las ocho especies principales, según el producto de cobertura y frecuencia, de cada una de las tres unidades de vegetación de la RECU-CN (Humedal, Bosque y Pastizal con arboledas). 


\section{DISCUSIÓN Y CONCLUSIONES}

\section{Riqueza de plantas vasculares de la RECU-CN}

En este trabajo se describió la vegetación de la Reserva Ecológica Ciudad Universitaria - Costanera Norte (RECU-CN), encontrándose una riqueza global de 154 especies, las cuales representan el $65 \%$ de las especies totales que habría en el área de estudio según la estimación realizada (238 especies). Esta diferencia puede deberse a que el muestreo se realizó durante la temporada de invierno, pudiendo resultar en una subestimación de especies al pasar desapercibidas aquellas especies anuales que podrían ser reconocidas en primavera y verano. La subestimación tampoco resulta sorpresiva ya que durante la realización del trabajo de campo se observaron especies que no se registraron dentro de ninguna parcela, como por ejemplo Pontederia cordata y Enterolobium contortisiliquum. A su vez, el valor estimado de especies totales del área fue de 238 , número cercano a lo registrado colectivamente y de forma histórica para el área, donde se alcanzó un total de 263 especies (Sirolli et al., 2018). De las 58 familias registradas, aquellas con más representantes resultaron ser Poaceae y Asteraceae, lo cual concuerda con lo registrado por Burkart (1957) para el delta del río Paraná y por Faggi \& Cagnoni (1990) para la costa de la ciudad de Buenos Aires.

En comparación con otrasáreas naturales costeras cercanas, relevadas con una metodología similar (Faggi \& Cagnoni, 1987; Kalesnik et al., 2005), la riqueza global registrada en la RECU-CN fue intermedia respecto a las 104 especies registradas en el Refugio Natural Educativo Ribera Norte (RN) y a las 242 especies registradas en la Reserva Ecológica Costanera Sur (RECS). Considerando la superficie total de estas tres áreas $(23,10$ y 350 ha respectivamente), la riqueza de plantas vasculares de la RECU-CN estuvo en el rango de lo esperado según la curva clásica de relación entre número de especies y área (Arrhenius, 1921). Por el contrario, el porcentaje de especies exóticas registradas en la RECU-CN (42\%) fue mucho mayor que en las otras dos áreas mencionadas (RN 14\%, RECS 27\%). Dicho porcentaje guardó la misma relación que el porcentaje de sustrato antrópico constituido por la península en la RECU-CN, por los terraplenes en la RECS y casi nula en el RN (Faggi \& Cagnoni, 1987, 1990; Kalesnik et al., 2005; Sirolli et al., 2018).
Si se considera que en las tres áreas se encontró mayor proporción de exóticas en dichos sustratos, que coinciden con las posiciones topográficamente más altas y transitadas, entonces los resultados son consistentes con que la actividad humana favorece la colonización por especies exóticas generando condiciones más secas en contextos ribereños y ayudando a la dispersión de especies exóticas, especialmente aquellas de ambientes terrestres (Catford et al., 2011; Jansen et al., 2011).

\section{Unidades de vegetación y comunidades de la RECU-CN}

La vegetación de la RECU-CN se ordenó a lo largo de dos gradientes principales: inundabilidad, creciente de izquierda a derecha, y antropización, creciente de derecha a izquierda (Fig. 2). Ambos factores son considerados estructuradores de la vegetación de suma relevancia en contextos ribereños (Webb et al., 2012; Osterkamp \& Hupp, 2010; You et al., 2015). Este resultado se reflejó no sólo a escala de las tres unidades de vegetación sino también dentro de las mismas, donde se diferenciaron comunidades de especies más o menos tolerantes al anegamiento, por ejemplo dentro del Humedal se diferenciaron el Canutillar de Louisiella elephantipes del Verdolagal de Ludwigia sp. y, dentro del Bosque, el Bosque de ceibo y ligustrina versus el Bosque de ligustro y paraíso. En un extremo, los sectores más bajos e inundables coinciden con los sectores menos transitados y disturbados por el ser humano y, en el otro, los sectores más altos poseen un sustrato de origen artificial y mayores frecuencia e intensidad de disturbios antrópicos (Sirolli et al., 2018). Estas condiciones también explican las principales diferencias fisionómicas de las unidades de vegetación, que se intercalan espacialmente entre fisonomías herbáceas y arbóreas con relativo equilibrio en extensión.

Las comunidades registradas en la unidad de Humedal constituyen fisonomías y elementos ampliamente reconocidos en la bibliografía del río Paraná, su delta y la ribera platense (Burkart, 1957; Neiff, 1986; Cabrera \& Zardini, 1993; Kalesnik et al., 2005; Sabattini \& Lallana, 2007; Passarelli et al., 2014). Según dichos autores, Louisiella elephantipes es una especie de gramínea flotante asociada a corrientes bajas 
a moderadas que crece con gran desarrollo en márgenes, marismas, lagunas y otros ambientes similares a donde se la registró en la RECUCN. Por su lado, Ludwigia sp. es una herbácea arraigada que se describe creciendo en terrenos inundables internos y costeros, como se observó en la RECU-CN. Además, la parcela ubicada sobre la costa (233) presentó gran cobertura de Phyllanthus sellowianus, una especie característica del matorral ribereño (Burkart, 1957; Cabrera \& Zardini, 1993). A pesar de no haberse registrado dentro de las parcelas realizadas en la unidad de Humedal, durante el muestreo también se observaron espadañales de Zizaniopsis bonariensis, totorales de Typha latifolia y juncales de Schoenoplectus californicus, todas comunidades también características de la región (Burkart, 1957; Passarelli et al., 2014). Las especies exóticas fueron escasas en esta unidad, sin embargo, a pesar de haberse registrado con baja cobertura y frecuencia, es destacable mencionar la presencia de Iris pseudacorus debido a su gran capacidad invasora (Kandus \& Quintana, 2018; Chen, 2019). De esta forma, las comunidades registradas presentaron una combinación de especies muy similar a la descrita por bibliografía en los ambientes naturales y con casi nula presencia de especies exóticas, en contraposición con la hipótesis de trabajo planteada.

En cuanto a la unidad de vegetación de Bosque, a pesar de la alternancia de la especie dominante en el dosel, se destacó la abundancia de Ligustrum sinense en el estrato medio. Esta especie asiática constituye una invasora exitosa en la región, que se desarrolla en etapas tempranas a intermedias en la sucesión (Kalesnik et al., 2005; Kalesnik \& Aceñolaza, 2008; Sirolli \& Kalesnik, 2015). Entre las causas de dicho éxito se mencionan la dispersión por aves durante la colonización inicial del área, la gran producción de frutos y la formación de un importante banco de semillas y de plántulas (Montaldo, 1993; Kalesnik et al., 2013). A su vez, se observó una baja cobertura de especies nativas (18\%) que, a diferencia de las comunidades del Humedal, propone una composición novedosa en comparación con sus contrapartes descritas en la bibliografía para ambientes naturales de la región pudiéndose considerar, en consecuencia, neoecosistemas.
La comunidad de Bosque de ceibo y ligustrina fue la única que se asemejó a una de las formaciones que se describen en la bibliografía de la región: el ceibal (Burkart, 1957; Cabrera \& Zardini, 1993). El dosel de esta comunidad estuvo dominado por Erythrina crista-galli y se registraron algunos componentes en común como las enredaderas Passiflora caerulea y Cissus palmata. Sin embargo, el estrato medio dominado por Ligustrum sinense y la ausencia de un sotobosque de ciperáceas marcan una importante diferencia explicada, probablemente, por el rápido cambio topográfico impuesto por la península, que determina baja superficie inundable. Por su lado, las especies dominantes del dosel de la comunidad de Bosque de ligustro y paraíso representan dos de las invasoras más emblemáticas de la región (Söyrinki, 1991; Ghersa et al., 2002; Torresín et al., 2013; Franco et al., 2018). A diferencia de su congénere, $L$. lucidum es más tolerante a la sombra, aunque comparte su elevado éxito reproductivo, explicado en parte por el bajo daño foliar que presenta en esta región, donde no es nativa (Montaldo, 2000; Montti et al., 2016). En cuanto al Bosque de eucalipto y ligustrina, si bien en Argentina se mencionan algunas especies naturalizadas del género Eucalyptus (Scaramuzzino et al., 2019), su crecimiento espontáneo y capacidad de invasión son limitados. En este caso, probablemente se trate de ejemplares plantados, ya que actualmente poseen un patrón poblacional remanente, dada la ausencia de plántulas registrada durante los muestreos. Por su lado, Arundo donax, la especie que domina la comunidad de Cañaveral de caña de Castilla, también es una especie exótica naturalizada en la costa rioplatense que forma parches densos cerca de arroyos, caminos y vías férreas (Söyrinki, 1991; Passarelli et al., 2014). El hecho de que esta comunidad haya sido clasificada dentro de la unidad de Bosque responde a su ubicación transicional entre esta unidad y la de Pastizal con arboledas, en donde algunas ramas de ligustro, ligustrina y paraíso se registraron dentro de las parcelas de cañaveral. El distanciamiento de estas parcelas respecto a las otras (números 68 y 85 en la figura 2), indican una composición considerablemente diferente a ambas unidades. 
En la unidad de vegetación de Pastizal con arboledas predominaron comunidades fisonómicamente similares a la estepa graminosa que cubría la mayor parte de la región (Cabrera \& Zardini, 1993; Paruelo et al., 2007). Sin embargo, su composición fue notablemente diferente, dominando especies de gramíneas no nativas (Burkart et al., 2011; Andrade et al., 2018), por lo que pueden considerarse neoecosistemas, de forma similar a lo discutido para las comunidades de Bosque. Las comunidades se diferenciaron en función de sus especies acompañantes: Ricinus communis, Dipsacus fullonum y Trifolium repens. Se trata de especies exóticas invasoras que crecen en terrenos productivos, artificiales o recientemente disturbados (Söyrinki, 1991; Sirolli \& Kalesnik, 2011; Busso et al., 2013). La Arboleda de tipa tuvo presencia de gramíneas en su sotobosque, por lo que quedó clasificada junto a los pastizales aunque su composición se aproximó también a la unidad de Bosque (comunidad 7, Fig. 2). Su especie dominante, Tipuana tipu, es nativa del noroeste de Argentina y constituye una especie común en el arbolado de la ciudad, crece en forma subespontánea en la región y es considerada invasora en otros lugares donde no es nativa (Valla et al., 1999; Sampaio \& Schmidt, 2014; Shabani et al., 2020). Fuera de las parcelas de muestreo también se observaron arboledas dominadas por otras especies ya mencionadas para la unidad de Bosque, como Eucalyptus sp. Estas arboledas se corresponden con lo tratado por la FAO como árboles fuera del bosque (de Foresta et al., 2013).

En conclusión, a pesar de tratarse de un relevamiento invernal, los resultados permitieron registrar el $59 \%$ del total de especies registradas históricamente para el área (Sirolli et al., 2018) $\mathrm{y}$ distinguir que las unidades de vegetación características de la RECU-CN se distribuyen entre Humedal, Bosque y Pastizal con arboledas. Las superficies hormigonadas son considerables y también pudieron ser detectadas mediante el muestreo. Con mayor detalle, pudieron registrarse y caracterizarse algunas de las comunidades que pueden observarse en campo, encontrándose composiciones similares a las descritas en la bibliografía de la región sólo para la unidad de vegetación de Humedal, mientras que las comunidades de Bosque y de Pastizal con arboledas presentaron características propias de los neoecosistemas.
Por lo analizado en el presente trabajo, es relevante considerar en el manejo del área los regímenes de inundación y de disturbios antrópicos, inclusive teniendoencuentaquela ocurrencia dealgunos de estos últimos puede determinar la permanencia de la unidad de Pastizal con arboledas. Por lo tanto, se destaca como principales valores de conservación la coexistencia de unidades de vegetación fisonómicamente diversas y relativamente equilibradas en extensión, así como también, la presencia de comunidades y especies típicas de la región dentro de la unidad de Humedal de la RECU-CN. Por último, el enriquecimiento con especies nativas en la península de relleno aumentará considerablemente dicho valor de conservación (Dearborn \& Kark, 2010; Holl, 2020).

\section{AGRADECIMIENTOS}

Por su ayuda con las tareas de campo a: Adriel M. Magnetti, Ana L. Cao, Daniela Prina, Daniel Zanola, David C. Clauser, Joaquín I. Salas, Magdalena Salas y Sebastián Benítez. Por la lectura crítica y sugerencias, a Natalia Morandeira, María Piedad Cristiano, Sebastián Torrella y dos revisores/as anónimos/as. Apoyo financiero: Consejo Nacional de Investigaciones Científicas y Técnicas (CONICET), subsidio "PIP 11220150100404CO". La presente investigación se realizó en el marco del Diagnóstico de situación y recomendaciones para la implementación de la RECU$\mathrm{CN}$, que llevó a cabo la Facultad de Ciencias Exactas y Naturales de la Universidad de Buenos Aires (Sirolli et al., 2018). Se dedica el presente trabajo al Dr. Fabio A. Kalesnik (1966-2017), docente e investigador especialista en bosques y humedales del Delta del Paraná.

\section{BIBLIOGRAFÍA}

Andrade, B. O.; E. Marchesi, S. Burkart, R. B. Setubal, F. Lezama, S. Perelman, A. A. Schneider, R. Trevisan, G. E. Overbeck \& I. I. Boldrini. 2018. Vascular plant species richness and distribution in the Río de la Plata grasslands. Botanical Journal of the Linnean Society 188(3): 250-256. DOI: https://doi.org/10.1093/botlinnean/boy063

APN. 2010. Guía para la elaboración de planes de gestión de áreas protegidas. Dirección Nacional de Conservación de Áreas Protegidas. Buenos Aires: Administración de Parques Nacionales. 


\section{R. MELZI FIORENZA ET AL. Vegetación de la Reserva Ecológica Ciudad Universitaria}

Arrhenius, O. 1921. Species and area. Journal of Ecology 9(1), 95-99. DOI: https://doi.org/10.2307/2255763

Athor, J. 2012. Buenos Aires. La historia de su paisaje natural. Buenos Aires: Fundación de Historia Natural Félix de Azara.

Barbetti, R.; A. Ronchetti \& J. C. Chebez. 1985. Refugio Educativo de la Ribera Norte, partido de San Isidro, provincia de Buenos Aires. Buenos Aires: Fundación Vida Silvestre Argentina.

Brailovsky, A. E. 2018. Historia ecológica de la Ciudad de Buenos Aires. Buenos Aires: MAIPUE.

Bridgewater, P.; E. S. Higgs, R. J. Hobbs \& S. T. Jackson. 2011. Engaging with novel ecosystems. Frontiers in Ecology and the Environment 9(8): 423-423. DOI: https://doi. org/10.1890/1540-9295-9.8.423

Burkart, A. 1957. Ojeada sinóptica sobre la vegetación del Delta del Río Paraná. Darwiniana 11(3): 457-561.

Burkart, A. 1969. Flora ilustrada de Entre Ríos (Argentina). Tomo VI, Parte II. Gramíneas. La familia botánica de los pastos. Buenos Aires: Colección Científica del I.N.T.A.

Burkart, A. 1974. Flora ilustrada de Entre Ríos (Argentina). Parte VI. Dicotiledóneas Metaclamídeas (Gamopétalas) B: Rubiales, Cucurbitales, Campanulales (incluso Compuestas). Buenos Aires: Colección Científica del I.N.T.A.

Burkart, A. 1979. Flora ilustrada de Entre Ríos (Argentina). Parte V. Dicotiledóneas Metaclamídeas (Gamopétalas). A: Primulales, Plumbaginales, Ebenales, Contortales, Tubiflorales, Callitrichales, Plantaginales. Buenos Aires: Colección Científica del I.N.T.A.

Burkart, A. 1987. Flora ilustrada de Entre Ríos (Argentina). Parte III. Dicotiledóneas Arquiclamídeas. A: Salicales a Rosales (incluso Leguminosas). Buenos Aires: Colección Científica del I.N.T.A.

Burkart, R.; N. O. Bárbaro, R. O. Sánchez \& D. A. Gómez. 1999. Eco-regiones de la Argentina. Buenos Aires: Administración de Parques Nacionales.

Burkart, A. \& N. M. Bacigalupo. 2005. Flora ilustrada de Entre Ríos (Argentina). Parte IV. Dicotiledóneas Arquiclamídeas. B: Geraniales a Umbelliflorales. Buenos Aires: Colección Científica del I.N.T.A.

Burkart, S. E.; R. J. C. León, M. C. Conde \& S. B. Perelman. 2011. Plant species diversity in remnant grasslands on arable soils in the cropping Pampa. Plant Ecology 212(6): 10091024. DOI: https://doi.org/10.1007/s11258-010-9881-z

Busso, C. A.; D. J. Bentivegna \& O. A. Fernández. 2013. A review on invasive plants in rangelands of Argentina. Interciencia 38(2): 95-103.

Cabrera, A. L. \& G. Dawson. 1944. La selva marginal de Punta Lara en la ribera argentina del Río de La Plata. Revista del Museo de La Plata (nueva serie), sección Botánica 5: 267-382.

Cabrera, A. L. (dir.). 1963. Flora de la Provincia de Buenos Aires, Parte VI. Compuestas. Buenos Aires: INTA.
Cabrera, A. L. (dir.). 1965a. Flora de la Provincia de Buenos Aires, Parte V. Dicotiledóneas gamopétalas (Ericáceas a Caliceráceas). Buenos Aires: INTA.

Cabrera, A. L. (dir.). 1965b. Flora de la Provincia de Buenos Aires, Parte IV. Dicotiledóneas dialipétalas (Oxalidáceas a Umbeliferas). Buenos Aires: INTA.

Cabrera, A. L. (dir.). 1967. Flora de la Provincia de Buenos Aires, Parte III. Dicotiledóneas dialipétalas (Piperáceas a Leguminosas). Buenos Aires: INTA.

Cabrera,A. L. (dir.). 1968. Flora de la Provincia de Buenos Aires, Parte I. Pteridófitas, Gimnospermas y Monocotiledóneas (excepto Gramineas). Buenos Aires: INTA.

Cabrera, A. L. (dir.). 1970. Flora de la Provincia de Buenos Aires, Parte II. Gramineas. Buenos Aires: INTA.

Cabrera, A. L. \& E. M. Zardini. 1993. Manual de la flora de los alrededores de Buenos Aires, $2^{\mathrm{a}}$. edición. Buenos Aires: ACME S.A.

Capllonch, P.; D. Ortiz \& K. Soria. 2008. Importancia del litoral fluvial argentino como corredor migratorio de aves. INSUGEO, Miscelánea 17: 107-120.

Catford, J. A.; B. J. Downes, C. J. Gippel \& P. A. Vesk. 2011. Flow regulation reduces native plant cover and facilitates exotic invasion in riparian wetlands. Journal of Applied Ecology 48(2): 432-442. DOI: https://doi.org/10.1111/ j.1365-2664.2010.01945.x

Charrad, M.; N. Ghazzali, V. Boiteau \& A. Niknafs. 2014. NbClust: An R Package for Determining the Relevant Number of Clusters in a Data Set. Journal of Statistical Software 61(1): 1-36. DOI: https://doi.org/10.18637/jss.v061.i06

Chen, L. 2019. Invasive Plants in Coastal Wetlands: Patterns and Mechanisms. En An, S. \& J. T. A. Verhoeven (eds.), Wetlands: Ecosystem Services, Restoration and Wise Use, pp 97-128. Cham: Springer International Publishing. DOI: https://doi.org/10.1007/978-3-030-14861-4_5

Chouikhi, H., M. Charrad y N. Ghazzali. 2015. A comparison study of clustering validity indices. En Institute of Electrical and Electronics Engineers (ed.) 2015 Global Summit on Computer and Information Technology (GSCIT), pp 1-4. Sousse: IEEE. DOI: https://doi.org/10.1109/GSCIT.2015.7353330

de Foresta, H.; E. Somarriba Chávez, A. Temu, D. Boulanger, H. Feuily \& M. Gauthier. 2013. Towards the assessment of trees outside forests. Rome: Food and Agriculture Organization of the United Nations.

Dearborn, D. C. \& S. Kark. 2010. Motivations for conserving urban biodiversity. Conservation Biology 24(2): 432-440. DOI: https://doi.org/10.1111/j.1523-1739.2009.01328.x

Duan, H.; H. Zhang, Q. Huang, Y. Zhang, M. Hu, Y. Niu \& J. Zhu. 2016. Characterization and environmental impact analysis of sea land reclamation activities in China. Ocean y Coastal Management 130: 128-137. DOI: https://doi.org/10.1139/facets-2016-0058 
Duda, R. O. \& Hart, P. E. 1973. Pattern classification and scene analysis. New York: John Wiley \& Sons.

Faggi, A. M. \& M. Cagnoni. 1987. Parque Natural Costanera Sur: las comunidades vegetales. Parodiana 5(1): 135-159.

Faggi, A. M. \& M. Cagnoni. 1990. Flora del Parque Natural Costanera Sur. Parodiana 6: 49-66.

Franco, M. G.; M. C. P. Behr, M. Medina, C. Pérez, I. A. Mundo, J. M. Cellini \& M. F. Arturi. 2018. Talares del NE bonaerense con presencia de Ligustrum lucidum: Cambios en la estructura y la dinámica del bosque. Ecología Austral 28(3): 502-512.

Gaertner, M.; J. R. Wilson, M. W. Cadotte, J. S. MacIvor, R. D. Zenni \& D. M. Richardson. 2017. Non-native species in urban environments: patterns, processes, impacts and challenges. Biological Invasions 19: 3461-3469. DOI: https://doi.org/10.1007/s10530-017-1598-7

Gaston, K. J.; S. F. Jackson, L. Cantú-Salazar \& G. Cruz-Piñón. 2008. The ecological performance of protected areas. Annual review of ecology, evolution, and systematics 39: 93-113. DOI: https://doi.org/10.1146/annurev.ecolsys.39.110707.173529

Ghersa, C. M., E. de la Fuente, S. Suarez \& R. J. C. Leon. 2002. Woody species invasion in the Rolling Pampa grasslands, Argentina. Agriculture, ecosystems \& environment 88(3): 271278. DOI: https://doi.org/10.1016/S0167-8809(01)00209-2

Gray, C. L.; S. L. Hill, T. Newbold, L. N. Hudson, L. Börger, S. Contu, A. J. Hoskins, S. Ferrier, A. Purvis \& J. P. Scharlemann. 2016. Local biodiversity is higher inside than outside terrestrial protected areas worldwide. Nature Communications 7: 12306. DOI: https://doi.org/10.1038/ncomms12306

Guerrero, E. L.; F. L. Agnolin, P. Grilli, F. A. Suazo Lara, E. Boné, A. B Tenorio \& S. Nenda. 2017. Inventario de la fauna transportada por balsas de vegetación flotante en el Sistema fluvial del Río de La Plata. Revista del Museo Argentino de Ciencias Naturales 19(2): 177-183.

Hobbs, R. J.; S. Arico, J. Aronson, J. S. Baron, P. Bridgewater, V. A. Cramer, P. R. Epstein, J. J. Ewel, C. A. Klink, A. E. Lugo, D. Norton, D. Ojima, D. M. Richardson, E. W. Sanderson, F. Valladares, M. Vilà, R. Zamora \& Martin Zobel. 2006. Novel ecosystems: theoretical and management aspects of the new ecological world order. Global ecology and biogeography 15(1): 1-7. DOI: https://doi.org/10.1111/j.1466-822X.2006.00212.x

Holl, K. 2020. Primer of Ecological Restoration. Washington D. C.: Island Press.

Hsieh, T. C.; K. H. Ma \& A. Chao. 2016. iNEXT: an R package for rarefaction and extrapolation of species diversity (Hill numbers). Methods in Ecology and Evolution 7(12): 14511456. DOI: https://doi.org/10.1111/2041-210X.12613

Jansen, F.; J. Ewald \& S. Zerbe. 2011. Ecological preferences of alien plant species in North-Eastern Germany. Biological Invasions 13(12): 2691-2701. DOI: https://doi.org/10.1007/s10530-011-9939-4
Jensen, S. 1978. Influences of transformation of cover values on classification and ordination of lake vegetation. Vegetatio: 37(1): 19-31.

Kalesnik, F.; M. Cagnoni, P. Bertolini, R. Quintana, N. Madanes \& A. I. Malvárez. 2005. La vegetación del refugio educativo de la Ribera Norte, Provincia de Buenos Aires, Argentina. Invasión de especies exóticas. INSUGEO, Miscelánea 14: 139-150.

Kalesnik, F. \& P. Aceñolaza. 2008. Regional distribution of native and exotic species in levees of the lower delta of the Paraná river. Acta Scientiarum. Biological Sciences 30(4): 391-402. DOI: https://doi.org/10.4025/actascibiolsci.v30i4.5869

Kalesnik, F.; H. Sirolli \& M. Collantes. 2013. Seed bank composition in a secondary forest in the Lower Delta of the Paraná River (Argentina). Acta Botanica Brasilica 27(1): 40-49. DOI 10.1590/S0102-33062013000100006.

Kandus, P. \& R. D. Quintana. 2018. The Paraná River Delta. En Finlayson, C. M; G. R. Milton, R. C. Prentice \& N. C. Davidson (eds.), The Wetland Book, pp. 813-821. Netherlands: Springer. DOI: https://doi.org/10.1007/978-94-007-4001-3_232

Kowarik, I. 2011. Novel urban ecosystems, biodiversity, and conservation. Environmental pollution 159(8-9): 19741983. DOI: https://doi.org/10.1016/j.envpol.2011.02.022

Kreimer, A.; D. Kullock \& J. B. Valdes. 2001. Inundaciones en el área metropolitana de Buenos Aires. Washington, DC: The World Bank.

Kuhman, T. R.; S. M. Pearson \& M. G. Turner. 2010. Effects of land-use history and the contemporary landscape on nonnative plant invasion at local and regional scales in the forestdominated southern Appalachians. Landscape Ecology 25(9): 1433-1445. DOI: https://doi.org/10.1007/s10980-010-9500-3

Lugo, A. E. 2009. The emerging era of novel tropical forests. Biotropica 41(5): 589-591. DOI: https://doi.org/10.1111/ j.1744-7429.2009.00550.x

Marcomini, S. C. \& R. A. López. 2004. Generación de nuevos ecosistemas litorales por albardones de relleno en la costa de la ciudad de Buenos Aires. Revista de la Asociación Geológica Argentina 59(2): 261-272.

Milligan, G. W. \& M. C. Cooper. 1985. An examination of procedures for determining the number of clusters in a data set. Psychometrika 50(2): 159-179. DOI: https://doi. org/10.1007/BF02294245

Montaldo, N. H. 1993. Dispersión por aves y éxito reproductivo de dos especies de Ligustrum (Oleaceae) en un relicto de selva subtropical en la Argentina. Revista Chilena de Historia Natural 66: 75-85.

Montaldo, N. H. 2000. Éxito reproductivo de plantas ornitócoras en un relicto de selva subtropical en Argentina. Revista Chilena de Historia Natural 73(3): 511-524. DOI: https:// doi.org/10.4067/S0716-078X2000000300015 
Montti, L.; M. M. Ayup, R. Aragón, R. Qi, H. Ruan, R. Fernández, S. A. Casertano \& X. Zou. 2016. Herbivory and the success of Ligustrum lucidum: evidence from a comparison between native and novel ranges. Australian Journal of Botany 64(3): 181-192. DOI: https://doi.org/10.1071/BT15232

Morello, J. H.; G. D. Buzai, C. Baxendale, A. Rodríguez, S. D. Matteucci, R. E. Godagnone \& R. R. Casas. 2000. Urbanización y consumo de tierra fértil. Ciencia Hoy 10(55): 50-61.

Mueller-Dombois, D. \& H. Ellenberg. 1974. Aims and methods of vegetation ecology. New York: John Wiley \& Sons.

Nabel, P. E. \& R. M. Becerra Serial. 2008. Uso de técnicas geomáticas para el mapeo y caracterización de unidades geomórficas de la Ciudad de Buenos Aires (Argentina) mediante interpretación visual de parámetros morfométricos. Boletín de la Sociedad Geológica Mexicana 60(2): 173-185.

Naughton-Treves, L.; M. B. Holland \& K. Brandon. 2005. The role of protected areas in conserving biodiversity and sustaining local livelihoods. Annual Review of Environment and Resources 30: 219-252. DOI: https://doi.org/10.1146/ annurev.energy.30.050504.164507

Neiff, J. J. 1986. Aquatic plants of the Paraná system. En: Davies, D. B. R. \& D. K. F. Walker (eds.), The Ecology of River Systems, pp. 541-598. Netherlands: Springer. DOI: https://doi.org/10.1007/978-94-017-3290-1_11

Oksanen, J.; F. G. Blanchet, M. Friendly, R. Kindt, P. Legendre, D. McGlinn, P. R. Minchin, R. B. O’Hara, G. L. Simpson, P. Solymos, M. H. H. Stevens, E. Szoecs \& H. Wagner. 2019. Vegan: Community Ecology Package. R package version 2.5-6. http://cran.r-project.org/web/packages/vegan [consulta diciembre de 2019]

Osterkamp, W. R. \& C. R. Hupp. 2010. Fluvial processes and vegetation-glimpses of the past, the present, and perhaps the future. Geomorphology 116(3-4): 274-285. DOI: https:// doi.org/10.1016/j.geomorph.2009.11.018

Oyarzabal, M.; J. Clavijo, L. Oakley, F. Biganzoli, P. Tognetti, I. Barberis, H. M. Maturo, R. Aragón, P. I. Campanello, D. Prado, M. Oesterheld \& R. J. C. León. 2018. Unidades de vegetación de la Argentina. Ecología Austral 28(1): 40-63. DOI: https://doi.org/10.25260/EA.18.28.1.0.399

Paruelo, J. M.; E. G. Jobbágy, M. Oesterheld, R. A. Golluscio \& M. R. Aguiar. 2007. The Grasslands and Steppes of Patagonia and the Rio de la Plata Plains. En Veblen, T.; K. Young \& A. Orme (eds.), The physical geography of South America, pp. 232-248. New York: Oxford University Press.

Passarelli, L. M.; C. H. Rolleri, M. M. Ciciarelli, A. C. Dedomenici \& G. González. 2014. Flora vascular de humedales permanentes y transitorios bonaerenses (Buenos Aires, Argentina). Botanica Complutensis 38: 139-154. DOI: https://doi.org/10.5209/rev_BOCM.2014.v38.45782
Prezzi, C.; R. López, C. Vásquez, S. Marcomini \& S. Fazzito. 2011. Caracterización geofísica de zonas de relleno en la costa de la Ciudad de Buenos Aires: estudio piloto en Ciudad Universitaria. Geoacta 36(1): 77-95.

R Core Team. 2019. R: A language and environment for statistical computing. R Foundation for Statistical Computing, Vienna, Austria. https://www.R-project.org/

Sabattini, R. A. \& V. H. Lallana. 2007. Aquatic Macrophytes. En Iriondo, M. H.; J. C. Paggi \& M. J. Parma (eds.), The Middle Paraná River: Limnology of a Subtropical Wetland. Berlin: Springer. DOI: https://doi.org/10.1007/978-3-540-70624-3_8

Sampaio, A. B. \& I. B. Schmidt. 2014. Espécies exóticas invasoras em unidades de conservação federais do Brasil. Biodiversidade Brasileira 2: 32-49.

Santo Domingo Sartori, A.D. \& P. I. Picca. 2009. Listado preliminar de la flora vascular rioplatense del Parque Natural Ciudad Universitaria (Ciudad Autónoma de Buenos Aires, Argentina). Boletín de la Sociedad Argentina de Botánica 44(Supl.): 223.

Scaramuzzino, R. L.; C. O. D'Alfonso, J. F. Bardi, M. L. Gandini \& B. Lara. 2019. Eucalyptus viminalis Labill. (Myrtaceae) naturalizada en la República Argentina. Darwiniana, nueva serie 7(2): 342-351. DOI: https://doi.org/10.14522/ darwiniana.2019.72.843

Seastedt, T. R.; R. J. Hobbs \& K. N. Suding. 2008. Management of novel ecosystems: are novel approaches required? Frontiers in Ecology and the Environment 6(10): 547-553. DOI: https://doi.org/10.1890/070046

Sengupta, D.; R. Chen \& M. E. Meadows. 2018. Building beyond land: An overview of coastal land reclamation in 16 global megacities. Applied geography 90: 229-238. DOI: https://doi.org/10.1016/j.apgeog.2017.12.015

Servicio Meteorológico Nacional. 2020. Estadísticas climáticas normales 1981-2010 para el Aeroparque Jorge Newbery. https://www.smn.gob.ar [consulta en junio de 2020].

Shabani, F.; M. Ahmadi, L. Kumar, S. Solhjouy-fard, M. Shafapour Tehrany, F. Shabani, B. Kalantar \& A. Esmaeili. 2020. Invasive weed species' threats to global biodiversity: Future scenarios of changes in the number of invasive species in a changing climate. Ecological Indicators 116(106436): 1-10. DOI: https://doi.org/10.1016/j.ecolind.2020.106436

Sirolli, H. \& F. A. Kalesnik. 2011. Effects of fire on a forestgrassland ecotone in De La Plata River, Argentina. Plant Ecology 212(4): 689-700. DOI: https://doi.org/10.1007/ s11258-010-9855-1

Sirolli, H. \& F. A. Kalesnik. 2015. Composición, estructura y tendencia sucesional de un bosque de aliso de río (Tessaria integrifolia), en la Reserva Ecológica Costanera Sur, Ciudad Autónoma de Buenos Aires, Argentina. Historia Natural, Tercera Serie 5(1): 109-124. 
Sirolli, H.; A. De Miguel \& G. Chaparro. 2018. Diagnóstico de situación y recomendaciones para la implementación de la Reserva Ecológica Ciudad Universitaria - Costanera Norte. Buenos Aires: Facultad de Ciencias Exactas y Naturales, Universidad de Buenos Aires.

Söyrinki, N. 1991. On the alien flora of the province of Buenos Aires, Argentina. Annales Botanici Fennici 28(1): 59-79.

Torresín, J. A.; L. P. Zamboni, W. F. Sione, E. E. Rodríguez \& P. Aceñolaza. 2013. Modelado de la distribución espacial de árboles exóticos invasores (AEI) en el Parque Nacional PreDelta (Entre Ríos, Argentina). Multequina 22: 1-11.

UNEP-WCMC \& IUCN. 2016. Protected Planet report 2016. Cambridge UK and Gland, Switzerland: United Nations Environment Programme.

Valla, J. J.; L. S. Jankowski, D. Bazzano \& A. J. Hernández. 1999. Árboles Urbanos. En: Lahitte, H. B. \& J. A. Hurrel (eds.) Biota Rioplatense. Volumen $I V$. Buenos Aires: L. O. L. A.

van der Maarel, E. 2007. Transformation of coverabundance values for appropriate numerical treatment - Alternatives to the proposals by Podani. Journal of Vegetation Science 18(5): 767-770. DOI: https://doi. org/10.1111/j.1654-1103.2007.tb02592.x van der Maarel, E. \& J. Franklin. 2013. Vegetation ecology, $2^{\text {nd }}$ edition. Chichester: John Wiley \& Sons. DOI: https://doi. org/10.1002/9781118452592

Watson, J. E.; N. Dudley, D. B. Segan \& M. Hockings. 2014. The performance and potential of protected areas. Nature 515(7525): 67-73. DOI: https://doi.org/10.1038/nature13947

Webb, J. A.; E. M. Wallis \& M. J. Stewardson. 2012. A systematic review of published evidence linking wetland plants to water regime components. Aquatic Botany 103: 1-14. DOI: https://doi.org/10.1016/j.aquabot.2012.06.003

Yang, H. Y.; B. Chen, M. Barter, T. Piersma, C. F. Zhou,F. S. LI \& Z. W. Zhang. 2011. Impacts of tidal land reclamation in Bohai Bay, China: ongoing losses of critical Yellow Sea waterbird staging and wintering sites. Bird Conservation International 21(3): 241-259. DOI: https://doi.org/10.1017/ S0959270911000086

You, X.; J. Liu \& L. Zhang. 2015. Ecological modeling of riparian vegetation under disturbances: A review. Ecological Modelling 318: 293-300. DOI: https://doi.org/10.1016/j. ecolmodel.2015.07.002

Zuloaga, F. O.; M. J. Belgrano \& C. A. Zanotti. 2019. Actualización del Catálogo de las Plantas Vasculares del Cono Sur. Darwiniana, nueva serie 7(2): 208-278. 